\title{
The vegetation of the southern Langeberg, Cape Province. 3. The plant communities of the Bergfontein, Rooiwaterspruit and Phesantefontein areas
}

\author{
D.J. MCDONALD*
}

Keywords: classification, forest, fynbos, Langeberg, phytosociology

ABSTRACT

The fynbos shrubland communities of the southeastem Langeberg. Cape Province were analysed after sampling 97 quadrats in the Bergfontein, Rooiwaterspruit (Garcia's Forest Reserve) and Phesantefontein areas. Data were initially analysed using TWINSPAN and the resulting classification refined using Braun-Blanquet (BB) procedures. One Afromontane forest community and 16 fynbos communities are recognized and described. A hierarchical classification of the fynbos communities is proposed.

\section{LITTREKSEL}

Die fynbos-struikgemeenskappe van die suidoostelike Langeberge, Kaapprovinsie, is geanaliseer. 'n Totaal van 97 monsterpersele in die Bergfontein-, Rooiwaterspnit- (Garcia Staatsbos) en Phesantefontein-gebiede is ondersoek. Data is aanvanklik deur Twinspan geanaliseer en die resultaat d.m.v. Braun-Blanquet prosedures (BB) verfyn. Een Afro-montane woudgemeensk ap en 16 fynbosgemeenskappe word erken en beskryf. ' $n$ Hiërargiese klassifikasie van die fynbosgemeenskappe word voorgestel.

CONTENTS

Introduction

Study area

Location ..................... 240

Physiography and geology ............ 241

Soils ......................... 241

Climate ........................ 242

Methods . . . . . . . . . . . . . . . . . . . . . . 243

Vegetation ....................... 243

Afromontane Forest . . . . . . . . . . 244

Fynbos ....................... 244

1. Erica hispidula Shrublands ............. 244

I.1 Erica hispidula-Brunia alopecuroides Shrublands .....................244

1.2 Erica hispidula-Restio inconspicuus Shrublands ....................... 249

1.2.1 Restio inconspicuus-Chondropetalum mucronatum Shrublands .............. 249

1.2.2 Restio inconspicuus-Selago serrata Shrublands ........................ 251

1.2.3 Restio inconspicuus-Erica melanthera Shrublands ................... 252

1.3 Erica hispidula-Tetraria bromoides Shrublands

1.3.1 Tetraria bromoides-Berzelia galpinii Shrublands

3.2 Tetraria bromoides-lschyrolepis hystrix

Shrublands ........................
3.2.1 Ischyrolepis hystrix-Phylica rubra Shrublands

3.2.2 Ischyrolepis hystrix-Phylica pinea Shrublands

\footnotetext{
* Conservation Biology Research Unit. National Botanical Institute. Pri vate $B$ ag X7. Claremont 7735

MS. received: $1992-11-02$
}

\subsubsection{Tetraria bromoides-Hypodiscus striatus} Shrublands .................... 254

1.3.4 Tetraria bromoides-Protea coronata Shrublands ..................... 255

1.4 Erica hispidula-Protea nitida Shrublands .. 256

2. Erica versicolor-Agathosma ovata Shrublands ..................... 257

3. Cullumia aculeata var. aculeata Shrublands . 257

3.1 Cullumia aculeata-Leucadendron eucalyptifolium Shrublands ............... 258

3.1.1 Leucadendron eucalyprifolium-Protea neriifolia Shrublands ............... 258

3.1.1.1 Protea neriifolia-Merxmuellera decora Shrublands ................ 258

3.1.1.2 Protea neriffolia-Erica anticularis Shrublands .................... 258

3.1.2 Leucadendron eucalyptifolium-Elegia filacea Shrublands ................. 259

3.2 Cullumia aculeata-Protea lorifolia Shrublands .................... 259

3.2.1 Protea lorifolia-Ficinia laciniata Shrublands .................... 260

3.2.2 Protea lorifolia-Leucospermum calligerum Shrublands . . . . . . . . . . . . . 260)

Discussion and conclusions ........... 261

Acknowledgements ................ 262

References .................... 262

Errata: The vegetation of the southern Langeberg. Cape Province. 2. .................... 263

\section{INTRODUCTION}

A survey of the vegetation of the southern Langeberg was undertaken to describe the plant communities of this previously poorly documented mountain range. The descriptions of plant communities presented here are based on samples taken on the third of three sample transects 


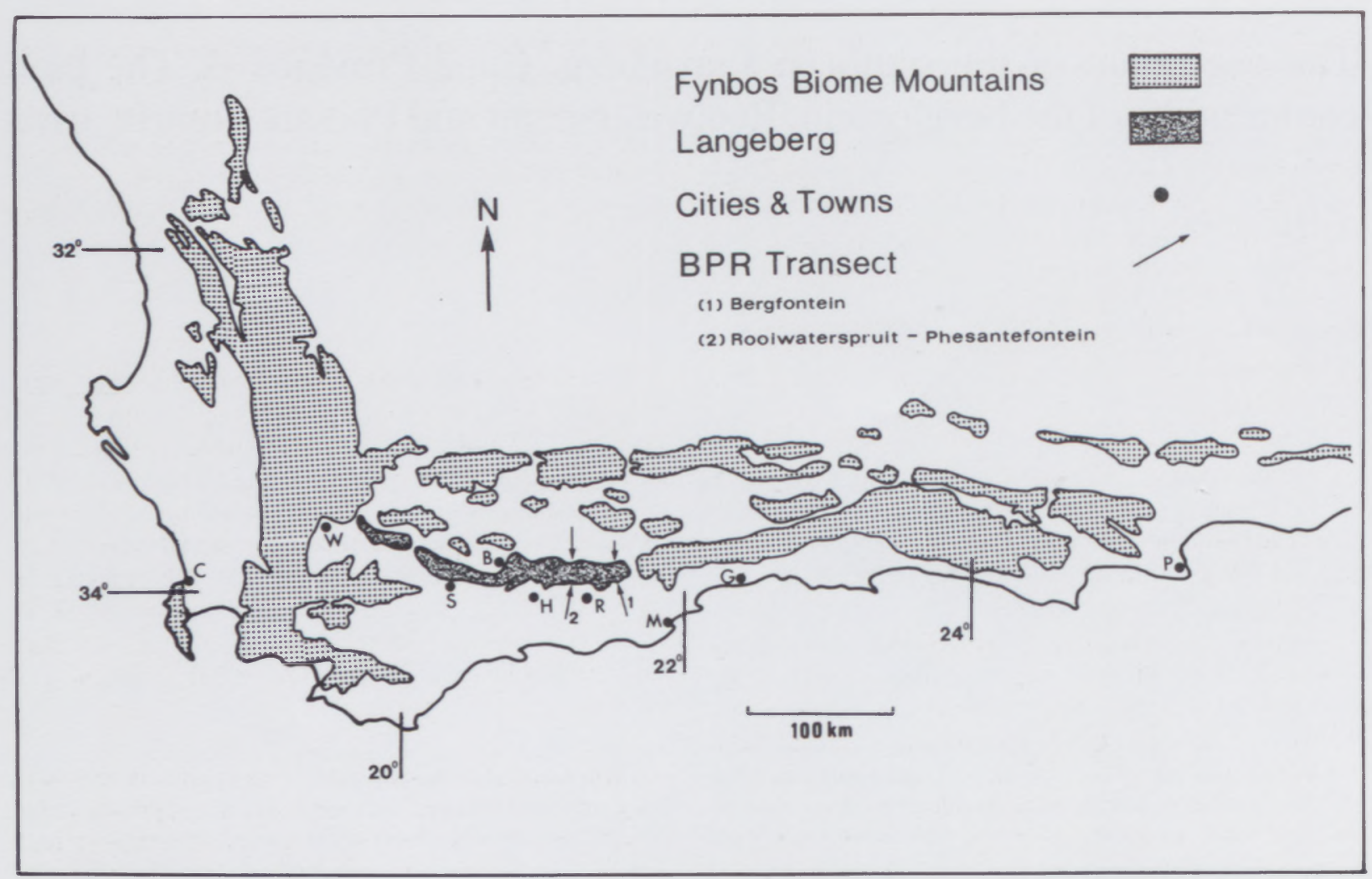

FIGURE 1.-Map of the mountains of the Fynbos Biome showing the position of the Langeberg and the location of the Bergfontein-RooiwaterspruitPhesantefontein areas. B. Barrydale; C, Cape Town; G, George; H, Heidelberg; M, Mossel Bay; P, Port Elizabeth; R, Riversdale; S, Swellendam and $\mathrm{W}$, Worcester.

straddling the Langeberg at intervals between Swellendam and the Gouritz River. Each transect is treated separately (McDonald 1993a \& b) but whereas the Marloth Nature Reserve (MNR) and Boosmansbos Wilderness Area (BWA) transects were more or less continuous from south to north, the third transect is a composite of samples from the Bergfontein area and the Rooiwaterspruit-Phesantefontein area (BRP) (Figure 1). This presented certain problems with respect to data analysis and interpretation which are discussed below.

\section{STUDY AREA}

\section{Location}

Numerous extensive wildfires have occurred on the Langeberg between Garcia's Pass and the Gouritz River in the past ten years (C. Martens pers. comm.). The fynbos vegetation on this part of the Langeberg is therefore almost all in a juvenile phase ( $<10$ years) (Kruger 1979). Little choice was left in finding mature fynbos along a continuous transect for sampling vegetation on the third transect over the southern Langeberg. A transect which satisfied most logistic and sampling criteria was selected in the Bergfontein area, $33^{\circ} 58^{\prime} \mathrm{S}, 21^{\circ} 33^{\prime} \mathrm{E}$. This area lies northeast of Riversdale, approximately midway between Garcia's Pass and the Gouritz River and is in the most easterly zone of the Langeberg. One negative feature was that the vegetation at Bergfontein was seven years old. However, despite the structural immaturity of the fynbos communities, the vegetation was in the 'maturing' successional phase (Kruger 1979) with the non-ephemeral species well established. It was therefore accepted that the floristic composition would reflect the 'mature' phase adequately and sampling could proceed.
The boundaries of the study area at Bergfontein followed the boundary between State Forest land and private agricultural land. The northern extreme of the designated transect extended onto private land where the vegetation was not disturbed by agriculture.

The Bergfontein transect followed the bridle path (Muir 1929) and sampling was largely confined to southand southwest-facing slopes east of Witelsrivier. Four sample plots were located west of Witelsrivier opposite the western end of Koksposberg to accommodate the Erica hispidula-Protea nitida Shrublands not found in the remainder of the study area.

Shortly after 74 relevés had been recorded on the southern slopes of the Bergfontein transect, a wildfire destroyed all the vegetation on the transect. Therefore alternative sites with vegetation and habitat as similar as possible to the vegetation on the northern slopes and highaltitude south-facing slopes had to be found. Since no suitable sites were available east of Garcia's Pass, alternative sites were chosen west of Garcia's Pass above Rooiwaterspruit (high-altitude south-facing slopes) and above Phesantefontein (north-facing slopes) $33^{\circ} 57^{\prime} \mathrm{S}, 21^{\circ} 9^{\prime} \mathrm{E}$.

At Rooiwaterspruit, which falls within the Garcia's Forest Reserve, four relevés (301-304) were recorded in vegetation on the Cedarberg Formation shaleband east of Stinkhoutbos. One plot (297) was located on the western side of the watershed between the headwaters of Rooiwaterspruit and Korinte River and three plots (298300 ) were located on the steep, high-altitude south-facing slopes of the unnamed peak immediately west of Sleeping 
Beauty (near trigonometric beacon 48). This latter peak is referred to as Korinteberg.

Above Phesantefontein, eight plots (286-293) were located in seven-year-old veld on the mid-altitude north-facing slopes east of Aasvoëlkrans (Garcia's Forest Reserve) and a further six (282-285, $295 \& 296)$ in mature veld ( $>20$ years) on the lower northern foothills of the Langeberg (Phesantefontein Farm).

\section{Physiography and geology}

\section{Bergfontein}

The south slopes of the Langeberg above Bergfontein are not as steep as the south slopes of the range further west. At the forefront of the mountain is Koksposberg, a prominent, isolated, low ridge-like hill or 'koppie'. This hill and others similar to it, to the west and east, ranging in altitude from 457-727 $\mathrm{m}$ and situated between the Kaffirkuils and Gouritz Rivers, are the remnants of the Peninsula Formation sandstone which is much more prominent further west. South of Koksposberg is a silcrete-capped mesa which supports fynbos. This area was excluded from the study since it fell outside the southern boundary of the transect and has been disturbed by agricultural practices. Immediately north of Koksposberg is the Cedarberg Formation shaleband which separates the Peninsula Formation sandstone from the Nardouw Subgroup sandstone. There is no deeply incised intermontane valley following the shaleband as there is in other parts of the Langeberg e.g. in BWA (McDonald 1993a). North of the shaleband is another 'koppie', of higher altitude than Koksposberg consisting of Nardouw Subgroup sandstone. Further north is Witelsberg which is a massive block of Nardouw Subgroup sandstone with steep south and north sides but with an expansive 'flat' top with shallow gradient from the west to the peak at $1166 \mathrm{~m}$ at the eastern end.

Below the steep north slopes of Witelsberg is a plateau extending from west of Witelsberg to Waboomsrivier. North of the plateau is a dissected sandstone ridge which gives way to arid sandstone slopes. They in turn make contact with the Bokkeveld Group shales of the Little Karoo.

\section{Rooiwaterspruit and Phesantefontein}

In the Garcia's Forest Reserve west of Garcia's Pass, the Peninsula Formation sandstone forms massive peaks on the southern side of the mountain range. Sleeping Beauty $(1343 \mathrm{~m})$ is the most prominent peak with Korinteberg $(1330 \mathrm{~m})$ to the west and lower peaks further westwards towards Gysmanshoek. Behind the peaks lie two incised valleys where streams have eroded the shales of the Cedarberg Formation. North of the shaleband lies the Nardouw Subgroup sandstone with Aasvoëlkrans west of Stinkhoutbos reaching $1341 \mathrm{~m}$ and another prominent peak east of Stinkhoutbos with a height of $1330 \mathrm{~m}$, referred to here as Phesanteberg.

Below the southern slopes of the mountain the Peninsula Formation sandstones make contact with silcretecapped plateau-like mesas which in turn are dissected by numerous watercourses. The high northern slopes of Nardouw Subgroup sandstone grade at a moderate angle to the undulating foothills at Phesantefontein. The foothills consist of sandstone overburden and the remains of a once extensive silcrete-capped landscape. Remnant mesas are encountered extending well into the Little Karoo (Figure 2).

\section{Soils}

The soils of the Bergfontein and RooiwaterspruitPhesantefontein areas of the Langeberg are the same as those of the MNR and BWA (McDonald 1993a \& b) at the level of 'form' (SCWG 1991). The climate of the three areas is similar (see below) as is the geology. Topographical variation and changes in parent material therefore account for most differences between the soils encountered on the BRP 'transect' and those of BWA and MNR. A description of the soil forms found on the southern Langeberg is given in McDonald (1993a); a summary of soil forms found on the BRP 'transect' follows:

Champagne Form soils are found at sites where there is a deep ( $>200 \mathrm{~mm}$ ) accumulation of organic material. On the BRP transect such sites are found mainly at high altitude on cool, moist south-facing slopes e.g. on Korinteberg. These soils also occur at lower elevations often in depressions where plant remains collect.

The catena of 'non-organic' soils derived from sandstone parent material in the study area include Houwhoek, Cartref, Glenrosa and Mispah Forms. Houwhoek and Cartref Form soils are closely related. The characteristics they share are an orthic A-horizon and presence of an E-horizon. They differ in that Houwhoek Form has a podzolised B-horizon overlying saprolite, whereas Cartref Form has a lithocutanic B-horizon. Glenrosa Form is similar to Cartref but lacks the eluviated E-horizon of the former. Mispah Form in turn is similar to Glenrosa Form, but here the orthic A-horizon overlies hard rock as opposed to the lithocutanic B-horizon of Glenrosa Form.

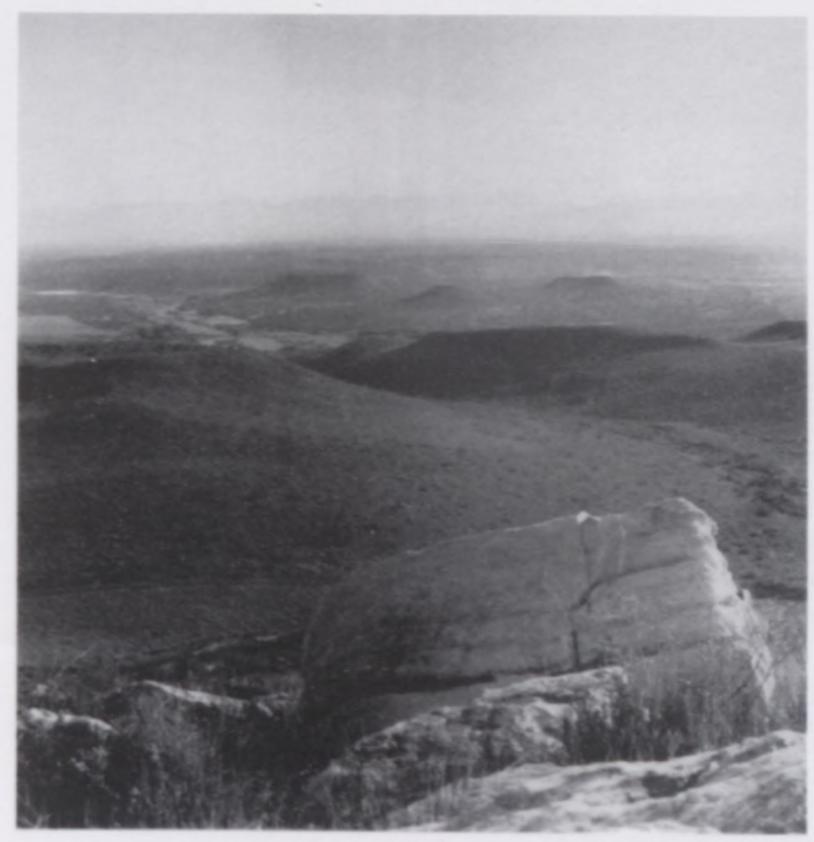

FIGURE 2.- Silcrete mesas on the north flank of the Langeberg, extending into the Little Karoo, at Phesantefontein. 
Clovelly Form soils which have an orthic A-horizon over a yellow-brown apedal B-horizon are found in two situations derived from different parent materials: 1 , on the Cedarberg Formation shale both at Bergfontein and at Rooiwaterspruit, behind Korinteberg: and 2. where there is accumulation of sand derived from Nardouw Subgroup sandstones, on dry north-facing slopes where leaching is limited.

Oakleaf Form soils which have limited extent in BWA (McDonald 1993a) but which were not identified in MNR occur on the lower south slopes at Bergfontein, below Koksposberg and on the west side of Witelsrivier. These soils which result from mixing of shale and sandstone have an orthic A-horizon and a reddish brown neocutanic B-horizon.

\section{Climate}

Characteristics of the climate of the BPR "transect" are similar to those described by McDonald (1993a \& b) for BWA and MNR. The southern Langeberg falls within a uniform bioclimatic zone, transitional between the winter rainfall region in the west and the year-round rainfall region in the east. The major climatic events which affect the weather of the southern Cape coast and the coastal mountains are the passage of cold fronts advancing from west to east, coastal lows and cutoff lows (Fuggle 1981; Van Heerden \& Hurry 1987). A Walter-Lieth climate diagram for Riversdale (Figure 3A) gives an approximation of the climate of the lower south slopes of the Langeberg in the Riversdale-Gouritz River District.

\section{Winds}

The direction of surface winds affecting the southern Langeberg is dependent on season with summer winds blowing mainly onshore and winter winds mainly offshore. Calm periods occur approximately one-third of the time in both summer and winter (Schulze 1965).

Extreme fire-hazard conditions prevail when hot, dry berg winds occur in the southem Cape, mainly in winter (Van Wilgen 1984). They arise from air subsiding in response to pressure gradients between an established anticyclone and an advancing depression (Fuggle 1981). The fire which swept through the Bergfontein area in May 1991 occurred in such conditions.

\section{Precipitation}

Precipitation on the Langeberg is from rain. mist and snow. Snowfalls occur once or twice each winter mainly on the high peaks and ridges and do not persist. Mist is a year-round phenomenon, usually occurring at elevations from $1000 \mathrm{~m}$ upwards. Rainfall occurs throughout the year with peaks in autumn (April) and spring (October). It may occur from unstable prefrontal conditions but is most often associated with postfrontal events. Eastwardmoving cold fronts are usually followed by a 'ridging-in' by the South Atlantic anticyclone behind the fronts. Air movement over the warm Agulhas Current results in onshore advection of cool moist air (Cowling 1984: Preston-Whyte \& Tyson 1988). Orographic rain occurs when this moist air strikes the coastal mountains.

\section{RIVERSDALE (122 m)}

'C [14]

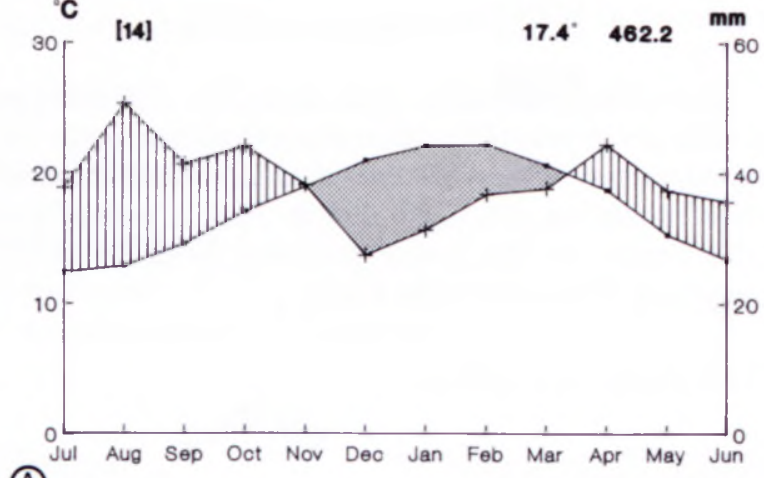

(A)

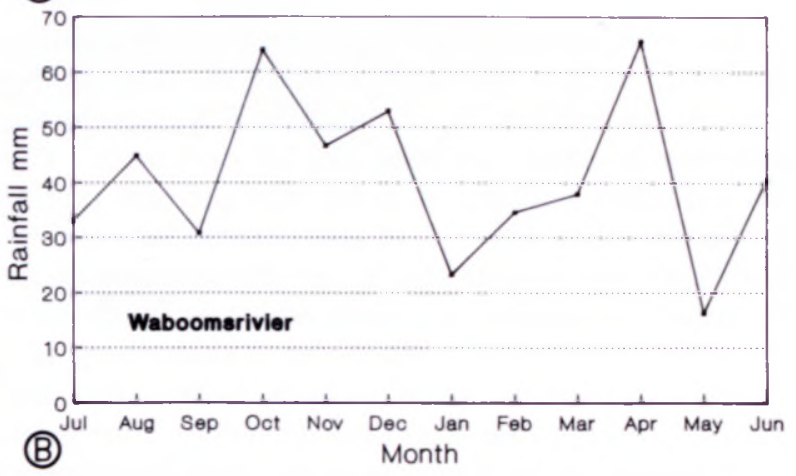

FIGURE 3.-A, climate diagram for Riversdale which approximates the climate of the lower south slopes of the Langeberg in the Riversdale-Gouritz. River District: B, monthly rainfall (19841991 ) at W aboomsrivier on the north side of Witelsberg, showing peaks in April (autumn) and October (spring).

The high peaks in the MNR at Swellendam and in BWA receive an estimated $12(0)-14(x) \mathrm{mm}$ rainfall per annum. Proceeding eastwards, Kanetberg receives $5(x)$ $6(0) \mathrm{mm}$. Aasvoëlkrans $6(0)-7()() \mathrm{mm}$, Korinteberg 7(0)$8(0) \mathrm{mm}$ and Sleeping Beauty $8(0)-9()() \mathrm{mm}$ rainfall p.a. East of Garcia's Pass the peaks receive from $8(0)-9()() \mathrm{mm}$ rainfall but the Bergfontein area (including Witelsberg south slopes) is much drier, receiving $6(0)-7(0) \mathrm{mm}$ rainfall p.a. (Dent et al. 1987). This gradient is opposite to that reflected in the mean annual rainfall for Heidelberg $(378 \mathrm{~mm})$ and Riversdale (426 mm) (Rebelo et al. 1991). The difference is attributed to the effect of orographic rainfall on the Langeberg; the more westerly higher altitude peaks receive more rainfall than the somewhat lower eastern part of the Langeberg close to the Gouritz. River.

The north slopes of Witelsberg are in a rain shadow and are consequently much drier than the southem slopes. The mean annual precipitation for a seven-year period (1984-1990) at Waboomsrivier at the northern base of Witelsberg was $496 \mathrm{~mm}$ (P.E. Wadman pers. comm.); seasonal distribution of the rainfall is as shown in Figure 3B. Rooiwaterspruit and Korinteberg receive a mean annual precipitation of $7(0)-8()) \mathrm{mm}$, whereas the upper north slopes above Phesantefontein (east of Aasvoélkrans) receive $5(0)-6(x) \mathrm{mm}$ and the lower north slopes $3(0)-500$ $\mathrm{mm}$ depending on locality (Dent et al. 1987).

\section{Temperature}

No temperature data are available for the study area: a situation commonplace in the Cape mountains where there are few weather stations (Bond 1981: Fuggle \& Ash- 
ton 1979; Fuggle 1981). Extrapolation of temperature data from 'lowland" weather stations to montane situations has been done (Van Wilgen 1984) but this does not reflect the true montane temperature regime in most cases. The closest temperature recording station to the study area is at Riversdale. Temperatures recorded here may approximate those experienced on the lower south slopes of the Langeberg. Therefore, if the environmental lapse rate of $0.6^{\circ} \mathrm{C} / 100 \mathrm{~m}$ (Cowling 1984) is used to predict montane temperatures, the high altitude south-facing slopes of Witelsberg at $1150 \mathrm{~m}$ are predicted to have temperatures $6.26^{\circ} \mathrm{C}$ lower than Riversdale at $106 \mathrm{~m}$.

\section{Solar radiation}

Incoming radiation may be measured directly (Morris 1981), which is cumbersome in mountainous terrain, or derived from sunshine duration. No data are published for sunshine duration on the Langeberg. Bond (1981) used Swift's (1976) algorithm to calculate potential radiation for a range of slopes and aspects for the $33^{\circ} 30^{\prime} \mathrm{S}$ latitude which is roughly equivalent to the latitude for the southern Langeberg. Bond (1981) found that potential radiation on the Swartberg and Outeniqua Mountains is relatively similar on all aspects and slopes in summer, with marked differences between north and south aspects, particularly on steep slopes, in winter. This holds for the Langeberg as well.

\section{METHODS}

Ninety-seven $5 \times 10 \mathrm{~m}$ quadrats (McDonald 1983 . 1988, 1993a \& b; Campbell 1985: Boucher 1987) were sampled on a "composite transect' over the Langeberg in the Bergfontein, Roojwaterspruit and Phesantefontein areas. No stratification of the study area was undertaken since no suitable aerial photography was available. Plots were therefore subjectively placed at sites representative of major landscape features and vegetation communities.

Floristic, structural and environmental data were collected from each sample plot. Permanently recognizable species were recorded using the Braun-Blanquet coverabundance scale (Mueller-Dombois \& Ellenberg 1974 Werger 1974: Westhoff \& Van der Maarel 1973). The midpoint of the $\mathrm{BB}$ values given as percentage cover is as follows: $5=87.5 \% ; 4=62.5 \%: 3=37.5 \%: 2=15.0 \%$; $1=2.5 \% ;+=0.1 \%: R=$ value ignored. Ephemeral geophytes and annuals were noted in each relevé but were not used in analyses and descriptions of communities. A border zone of $1.5 \mathrm{~m}$ from the perimeter of each plot was searched for any species not found in the marked plot. Species occurring outside the plot are represented by ' $O$ ' in the phytosociological tables. Vegetation structure was measured by estimating the projected canopy cover and height of the respective strata. Environmental variables recorded include altitude, aspect, slope, geology, soil form. drainage and estimated soil depth. Mean annual rainfall was estimated from isohyet maps prepared by Dent et al. (1987).

Samples were taken only in fynbos shrubland communities. Stinkhoutbos in the Rooiwaterspruit area is a welldeveloped stand of Afromontane Forest but was not formally sampled (see below).
Two-way Indicator Species Analysis-TWINSPAN (Hill 1979a) was used to obtain an initial tabular classification of the data. This was followed by successive refinement of the phytosociological tables following the Braun-Blanquet method (Mueller-Dombois \& Ellenberg 1974: Werger 1974) using the PCTables programs (Boucher pers. comm.). Detrended Correspondence AnalysisDECORANA Hill 1979b) was used to assess the relationship of the Erica versicolor-Agathosma ovata Shrublands to the other shrublands in the Bergfontein area (see below).

The plant communities are described in the order of the proposed hierarchical classification (see above). No syntaxonomic rank is assigned to any given community. Structural description follows the a priori system of Campbell et al. (1981).

The 'relationships' between communities described in this study and those described in other, previous studies of Mountain Fynbos were determined on the basis of floristic (mainly) and structural similarity. The similarities were determined from published descriptions and phytosociological tables. No rigid system was applied and the relationships serve merely as a guide for future synthesis of fynbos communities.

\section{VEGETATION}

The primary objective of a study such as this is to characterize the plant communities of a given area so that they may be repeatedly identified where they occur in the landscape. The early descriptions of the vegetation of the southeastern Langeberg by Muir (1929) in his treatment of the vegetation of the Riversdale area are highly informative but, apart from a broad classification, do not provide clear delineations of the fynbos communities. The fynbos vegetation sampled on the composite BPR 'transect' is classified into 16 communities: 10 at Bergfontein. two at Rooiwaterspruit and the remaining four at Phesantefontein. One Afromontane Forest community is recognized. The classification of the plant communities of the BPR transect is not complete since the scale of the study dictated that not all communities could be exhaustively sampled in the limited study area. Emphasis is on the sclerophyllous fynbos plant communities, since communities of this type make up the major part of the vegetation in the study area. Afromontane Forest communities are extremely limited in extent.

The vegetation of the south slopes of the Bergfontein area is mainly wet to mesic proteoid fynbos with a few isolated patches of trees in protected places. These trees such as Cumonia capensis are representative elements of Afromontane Forest. A well-developed stand of this forest type is located on an east-facing cliff of Perdeberg opposite Witelsberg. This forest is difficult to reach and fell outside the study area so it was not sampled. On the northem extreme of the Bergfontein transect, i.e. on the lower north slopes of Witelsberg. dry proteoid fynbos is encountered.

At Rooiwaterspruit, the south slopes also support wet proteoid and ericaceous fynbos with one well-developed patch of Afromontane Forest. Stinkhoutbos, in a moist 
kloof or ravine. The north-facing slopes above Phesantefontein have mesic proteoid to dry asteraceous fynbos communities.

During preparation of the phytosociological tables it was initially doubted whether relevés taken in mature or senescent vegetation (sensu Kruger 1979) would be satisfactorily accommodated, for reasons such as lack of differential species and overriding dominance of tall shrubs. It was interesting to note, however, that these relevés were appropriately placed in the tables and that they gave insights into the nature of the respective communities when they reach the mature and senescent phases (see 1.3.1 and 1.3.2.1 below).

\section{Afromontane Forest}

The description of Afromontane Forest is confined to the community found at Stinkhoutbos (Figure 4). No samples were taken in this forest which covers approximately 2 ha. A list of species confirms that it may be classified as the Cunonia capensis-Platylophus trifoliatus Subassociation (McKenzie 1978), also found in BWA (McDonald 1993a). As the colloquial name of the forest stand suggests, the stinkwood Ocotea bullata is common and co-dominant with Cunonia capensis and Platylophus trifoliatus in the canopy. Virgilia oroboides forms large trees mainly on the forest margins. Plectranthus fruticosus is the dominant understorey shrub.

\section{Fynbos}

\section{Erica hispidula Shrublands}

The role of Erica hispidula in linking the fynbos communities of the mesic to wet slopes of the southwestern and southern Cape mountains has been indicated by McDonald (1993a \& b). The south slopes of the Langeberg at Bergfontein and Rooiwaterspruit are no exception. Erica hispidula is found in all the fynbos communities at Bergfontein except in the Tetraria bromoides-Phylica

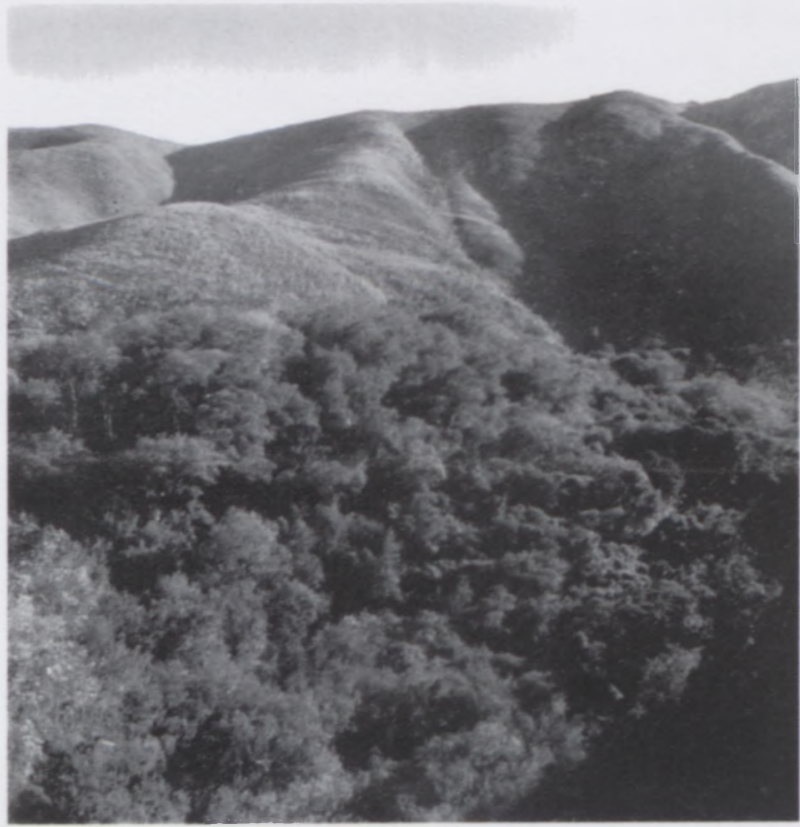

FIGURE 4.-Stinkhoutbos, a patch of Afromontane Forest in a secluded kloof at Rooiwaterspruit. pinea Shrublands and with little occurrence in the wet Erica hispidula-Brunia alopecuroides Shrubland sampled on Korinteberg. The Erica hispidula-Restio inconspicuus Shrublands include the very wet high altitude shrublands on the south-facing slopes of the peaks, as well as shrublands forming part of the mosaic of communities on the mid and lower south-facing slopes of the Bergfontein area

\subsection{Erica hispidula-Brunia alopecuroides Shrublands}

Differential species: Brunia alopecuroides, Erica albens, E. mucronata, E. regerminans, E. transparens, Hippia integrifolia, Helichrysum capense, Indigofera concava, Lobelia pubescens var. rotundifolia, Restio fragilis.

Dominant species: Anthochortus crinalis, Brunia alopecuroides, Spatalla parilis, Platycaulos compressus.

Structural formation: Low Closed Restioid Shrubland.

Relationships: Brunia alopecuroides-Restio bifidus Community (Kruger 1974); Subcommunity E2 of the Erica-Penaea Community (Glyphis et al. 1978); RestioHypolaena Subcommunity (H \& I) (Laidler et al. (1978); Ericoid-Restioid Zone Fynbos (Taylor 1978); Low Narrow-sclerophyllous Heathland (Kruger 1979); Simocheilus carneus-Restio anceps Community (Bond 1981); Wet Mountain Fynbos (Moll et al. 1984); Ruitersberg Wet Ericaceous Fynbos (Campbell 1985); Erica hispidula-Spatalla nubicola Shrublands and Restio inconspicuusAnthochortus crinalis Shrublands (McDonald 1993a): Anthochortus crinalis-Erica curviflora Shrublands (McDonald 1993b).

Owing to the fire in the Bergfontein area during this survey, sampling of the vegetation of the high altitude south-facing slopes of Witelsberg was not possible. As a substitute, four samples (297-300) were taken on similar slopes on Korinteberg. The community represented is equivalent to that found on Witelsberg prior to the fire (D.J. McDonald pers. obs.).

Moist air moving onshore from the southern Cape coast, together with mist and stratus cloud result in high orographic precipitation on the high altitude (above 1000 m) south-facing slopes. The high precipitation, low temperature and reduced insolation result in low rates of organic matter decay and consequent accumulation of deep layers of peat-like material. This deep acid peat $(\mathrm{pH} 3.0$ in $1 \mathrm{~mol} / 1 \mathrm{CaCl}_{2}$ ) with underlying Peninsula Formation sandstone, or Champagne Form soil, supports the Erica hispidula-Brunia alopecuroides Shrublands which are typical of these habitats (McDonald 1993a \& b).

The Erica hispidula-Brunia alopecuroides Shrublands on Korinteberg (Figure 5) are well differentiated floristically (Table 1) with dense stands of mid-high Brunia alopecuroides shrubs dominating the upper stratum. Spatalla nubicola (Proteaceae), a narrow endemic, which is found in the equivalent shrubland community in the BWA (McDonald 1993a) is replaced by the closely allied $S$. parilis on Korinteberg. Anthochortus crinalis (Restionaceae), a dense mat-restio, dominates the understorey.

A specimen of Erica dodii was found in plot 298 on Korinteberg, which is the first record of this species on 
TABLE 1. - Phytosociological table of the Erica hispidula Shrublands on the south slopes of the Bergfontein area, southern Langeberg

\begin{tabular}{|c|c|c|c|c|c|c|c|c|c|c|}
\hline & \multicolumn{10}{|c|}{ Community } \\
\hline & \multirow{2}{*}{$\frac{1.1}{4}$} & \multicolumn{3}{|c|}{1.2} & \multicolumn{5}{|c|}{1.3} & \multirow{2}{*}{$\frac{1.4}{j}$} \\
\hline & & B & c & 0 & E & $\mathrm{f}$ & 6 & $H$ & I & \\
\hline \multirow{4}{*}{ Altitude $(n)$} & \\
\hline & \multicolumn{10}{|c|}{1121.676588} \\
\hline & \multicolumn{10}{|c|}{2185,9160443} \\
\hline & \multicolumn{10}{|c|}{1271.7278086 .0504009122 .530218078872 .5534049804 .6859 .703844 .95690894955 .063594 .0336} \\
\hline Aspect $(*)$ & \multicolumn{10}{|c|}{$2122.1222222 .1122211221 .1112222221 \quad .1122222222 .2222 .321111 .3333 \quad 3233.222211 .11$} \\
\hline & \multicolumn{10}{|c|}{5810.7130025 .9952242440 .907573542875 .9904811290 .5296 .516636 .01401223843 .658836 .6009} \\
\hline & \multicolumn{10}{|c|}{4574.0002550 .0550100225 .586003201583 .5555000500 .3000 .084770 .95855190805 .066570 .5005} \\
\hline Slope $(")$ & \multirow{2}{*}{\multicolumn{10}{|c|}{ 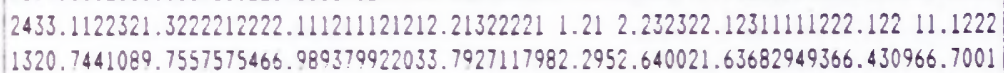 }} \\
\hline & & & & & & & & & & \\
\hline Releve number & \multirow{3}{*}{\multicolumn{10}{|c|}{$\begin{array}{l}2223 * 2222222 * 2222222222 * 222222222222 * 2222222222 * 3333 * 222222 * 22222222222 * 222222 * 2222 \\
9990 * 5566777 * 3335555777 * 223334445577 * 114444566 * 000 * * 233335 * 11122222466 * 111224 * 6666 \\
7890 * 8909346 * 1271267012 * 890890563457 * 231348053 * 1234 * 734560 * 78902346212 * 456157 * 5678\end{array}$}} \\
\hline & & & & & & & & & & \\
\hline & & & & & & & & & & \\
\hline
\end{tabular}

Differential species of the Erica hispidula--Brunia alopecuroides Shrublands (1.1)

Brunia alopecuroides thuno.

Spatalla parilis Salisb. ex knight

Erica regerminans $b$.

Erica albens L.

Erica mucronata Andr.

Erica transoarens Berg.

Hidpla integrifolia less.

Helichrysum capense Hilliard

Indigofera concava Harv.

Lobelia pubescens Dryand ex Ait. var. cotundifolia

Restio fragilis Esterhuysen

$\left|\begin{array}{c}5223 \\ 221 \\ 12+ \\ +++ \\ +1 R \\ R 1 \\ R++ \\ 0 R \\ R \\ R+ \\ R+ \\ R\end{array}\right|$

Differential species of the Restio inconspicuus--Chondrodetalun mucronatum Shrublands (1.2.1)

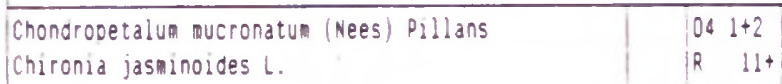

Species common to communities $1.1 \& 1.2 .1$

\begin{tabular}{|c|c|c|c|c|c|c|c|}
\hline Platycaulos compressus (Rottb.) Linder & R233.12 22222 & . & . & . & . & - & . \\
\hline 8laeria coccinea Klotzsch & $11.32213 \mathrm{R}+$ & R & . & . & . & . & . \\
\hline Epischoenus auadrangularis (Boeck.) C.B. Cl. & $++4+2+3$ & & . & . & . & - & - \\
\hline Anthochortus crinalis (Mast.) Linder & 444.2 & & . & . & . & . & . \\
\hline Platycaulos anceps (Mast.) Lincer & 4. & & . & & . & . & - \\
\hline Epischoenus cf. adnatus Levyns & 1. & & . & . & . & . & . \\
\hline Erica curviflora L. & 0 & & . & . & . & & . \\
\hline
\end{tabular}

Differential species of the Restio inconsoicuus--Selago serrata Shrublands (1.2.2)

Dthonna quinquedentata Thunb.

Selago serrata Berg.

Syncarpha vestita (L.) 8 . Nord.

Species common to communities $1.2 .1 \& 1.2 .2$

Pentaneris macrocalycina (Stevo.) Schweickerot

Centella lanata compton

Syncarpha eximia (L.) B. Nord.

Erica gloniflora Salisb. var. glomiflora

Berzelia burchellii ouenner

Gnidia opdositifolia l.

Os teospermun corynoosun $L$.

$\left|\begin{array}{ccc}+0 & +0 & \\ 0 & 0 & R \\ & +1\end{array}\right|$

Species common to communities 1.1. 1.2.1, 1.2.2 \& 1.2.3

Pentaschistis alouinensis (Steud.) Clayton

penaea cneorum Meerb. SubsD. ovata (Eckl. \& leyh.

ichrysithrix capensis L.

Erica coryata Andr.

Tetraria brevicaulis C.B. Cl.

Senecio cordifolius L. $\$$

Centella sessilis Adamson

Tetraria thernalis (L.) C.B. Cl.

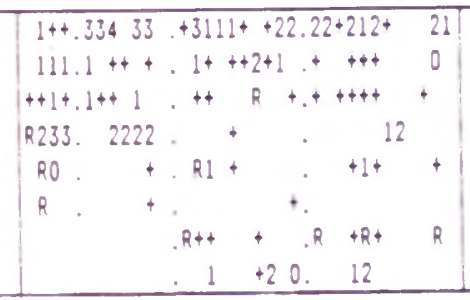

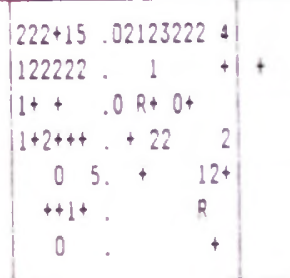


TABLE 1.-Phytosociological table of the Erica hispidula Shrublands on the south slopes of the Bergfontein area, southern Langeberg (continued)

Relevé number $12223 * 2222222 * 2222222222 * 222222222222 * 2222222222 * 3333 * 222222 * 22222222222 * 222222 * 2222$ $9990 * 5566777 * 3335555777 * 223334445577 * 1144444566 * 0000 * 233335 * 11122222466 * 111224 * 6666$ $7890 * 89009346 * 1271267012 * 890890563457 * 2313489534 * 1234 * 734560 * 78902346212 * 456157 * 5678$

Species common to communities $1.2 .1,1.2 .2,1.2 .3 \& 1.3 .1$

Restio inconscicuus Esterhuysen

Nevillez so. nov.

Berzelia galoinii Pillans

Agadanthus africanus (L.) Hoffing.

Edmondia sesanoides (L.) Hilliard

Ursinia trifida (Thunb.) N.E. 8 r.

Wahlenbergia fruticosa $V$. Brehm

Mairia crenata (Thunb.) Nees

iCullumia so. (unzdentified 237/17)

Senecio ourole if. underside (lanceolate)

Bodartia macrospatha Eak. Subso. anceos Strid

Erica seriohilfolia Salist.

Lachnaea so. nov.

Senecio ilicifolius (L.) Thunb.

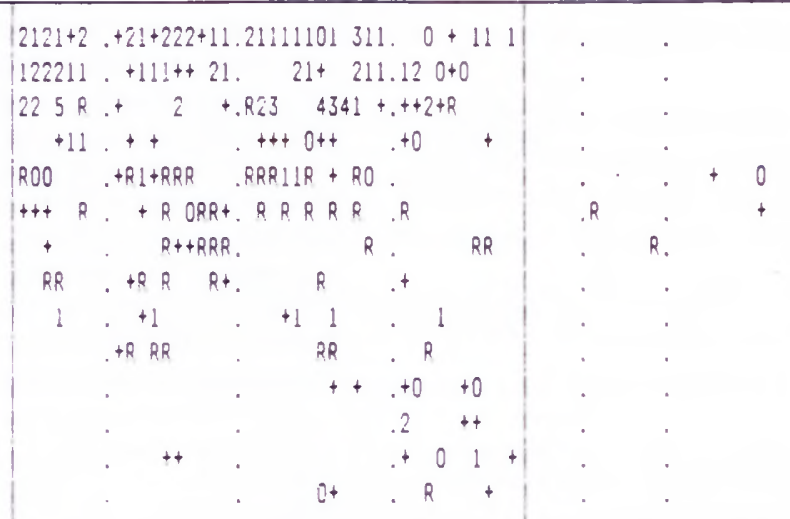

Soecies common to communities $1.1,1.2 .1,1.2 .2,1.2 .3 \& 1.3 .1$

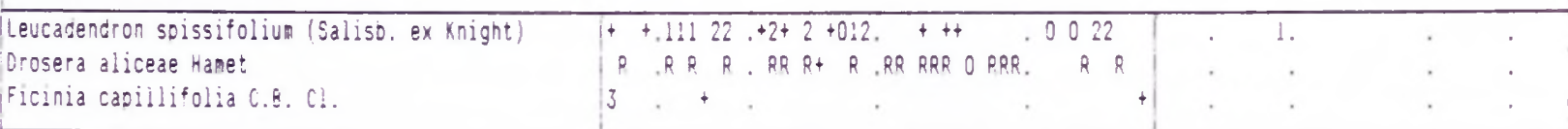

Differential soecies of the Ischyroledis hystrix--phylica rubra Shrublands $(1.3 .2 .1)$

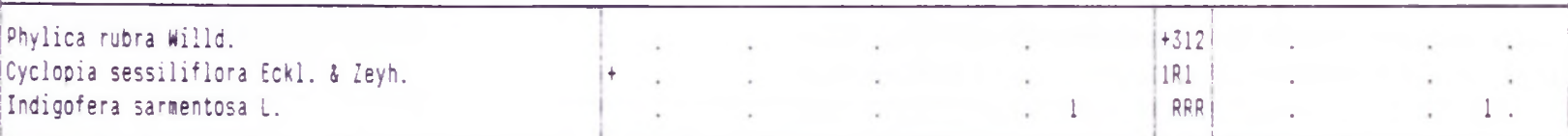

Differential species of the Ischyroledis hystrix... Phylica pinea Shrublands (1.3.2.2)

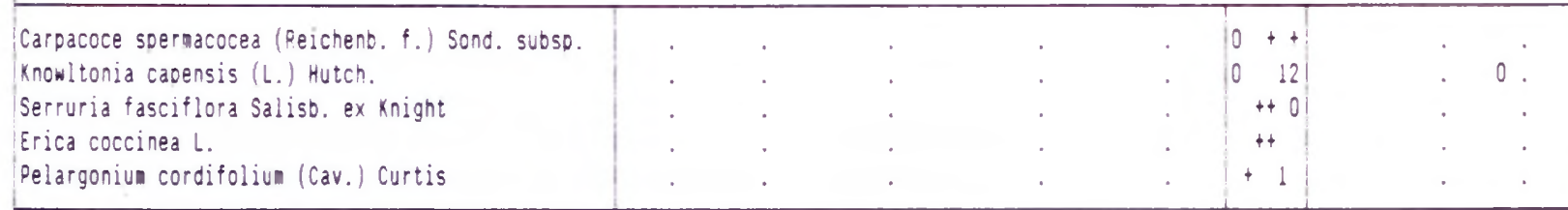

Soecies common to comnunities $1.3 .2 .1 \& 1.3 .2 .2$

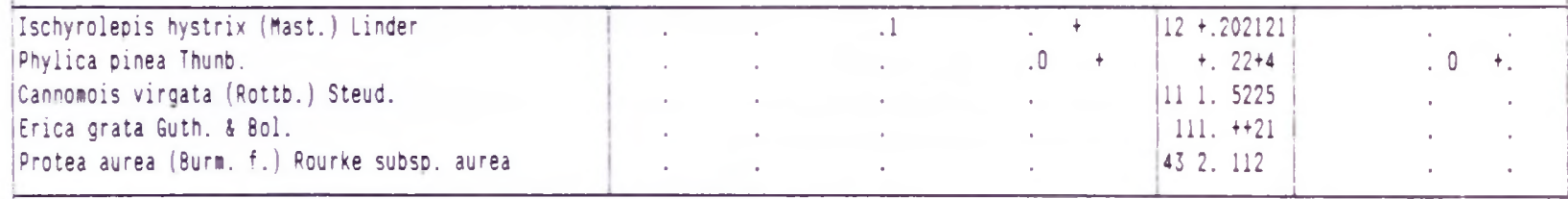

Species comnon to communities $1.2 .1,1.2 .2,1.2 .3,1.3 .1,1.3 .2 .1 \& 1.3 .2 .2$

Erica Denicilliformis Salisd.

Caloosis membranacea (Pillans) Linder

widdringtonia nodiflora (L.) Powrie

Laurophyllus capensis Thunb.

Elegia equisetacea (Mast.) Mast.

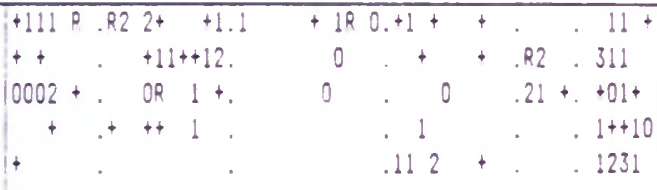

Differential species of the Tetraria bromoides--Hypodiscus striatus Shrublands (1.3.3)

Anomalanthus scooarius xlotzsch

Hypodiscus striatus (Kunth) Mast.

Heteropogon contortus (b.) Roen. \& Schult.

Diosma tenella willians

Peucedanum ferulaceum Thunb. var. ferulaceum

Polygala so. (unidentified 222/31)

Elegia galoinii M.E. 8r.

Species common to comunities $1.3 .2 .2 \$ 1.3 .3$

Osteospernum triquetrun L. $f^{\prime}$

Centella virgata (L.f.) Orude var. virgata

otholobiun so. (1) (unidentified) 
TABLE 1. - Phytosociological table of the Erica hispidula Shrublands on the south slopes of the Bergfontein area, southern Langeberg (continued)

$2223 * 2222222 * 2222222222 * 222222222222 * 2222222222 * 3333 * 222222 * 22222222222 * 222222 * 2222$ $19090 * 5566777 * 3335555777 * 223334445577 * 1144444566 * 0000 * 233335 * 11122222466 * 111224 * 6666$ $7890 * 8909346 * 1271267012 * 890890563457 * 2313489534 * 1234 * 734560 * 78902346212 * 456157 * 5678$

Species comnon to communities $1.2 .1,1.2 .2,1.2 .3,1.3 .1,1.3 .2 .1,1.3 .2 .2 \& 1.3 .3$

Merxmullera rufa (Nees) Conert

Erica triceps link

Hypodiscus aristatus (Thunb.) Krauss

Indigofera langebergensis L. Bol.

Acmadenia trigona Eckl. \& leyh.

Argyrolobiun filiforme Eckl. \& leyh.

Erica melanthera $L$.

Tetraria fasciata (Rottb.) C.B. Cl

Syncarpha paniculata (L.) B. Nord.

Pelargonium ternatum (L.f.) Jaca

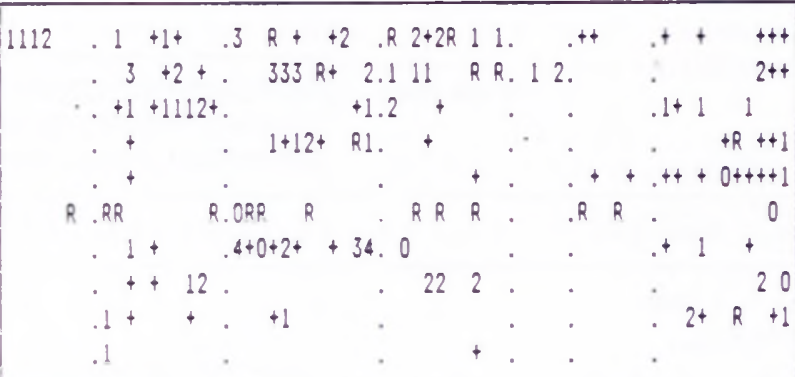

Differential species of the Tetraria bromoides--Protea coronata Shrublands (1.3.4)

Aleoidea Capensis R.A. Dyer

Protea coronata Lam.

Erica cf. zwartbergense

otholobium so. (2) (unidentified)

Species common to communities $1.3 .3 \& 1.3 .4$

Tetraria pillansii Levyns

Tetraria capillacea (Thunb.) C.B. Cl.

Species common to communities $1.3 .1,1.3 .2 .1,1.3 .2 .2,1.3 .3 \& 1.3 .4$

Tetraria bromoides (Lam.) Pfeiffer

Gnidia galpinil C.H. Wr.

Struthiola garciana C.H. Wrignt

Psoralea nonophylla (L.) C.H. Stirton

Thesium carinatum A. OC.

Clutia ericoides thunb, var. tenuis sond.

Sebaea stricta (E. Mey.) Gilg.

athrixia heteroohylla (Thunb.) Less 5ubso. heterod

Hyoodiscus albo-aristatus (Nees) Mas:.

Aspalathus hyproides lanlg.

iphylica purourea sond. var. floccosa pillans

Soecies common to communities ail comnunities excest $1.1 .1,1.1 .2$

Tetrarja crassa Levyns

Gerbera serrata (Thuno.) Druce

Berzeliz internedia schlechtd.

Psoralea cinnata $L$

Indigotera labellata Harv.

Stoebe alopecuroides (Lam.) Less.

Indigofera alopecuroides DC. var. minor

Tetrarla finbriolata (Nees) C.B. Cl.

Metalasia SD. (unidentified $219 / 26$ )

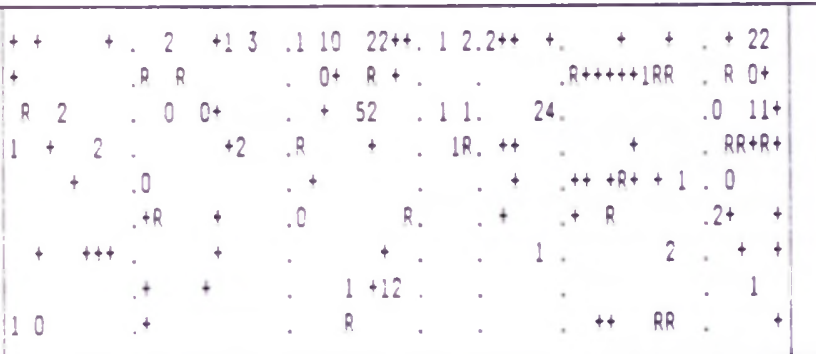

Species common to communities $1.2 .1,1.2 .2,1.2 .3,1.3 .1,1.3 .2 .1,1.3 .2 .2,1.3 .3 \& 1.3 .4$

Thamnochortus cinereus Linder

Enrharta dura Nees ex irin.

Leucadendron eucalyptifoliun Buek. ex Meisn

Staberona cernua (L.f.) Dur. \& Schinz.

Tetraria flexuosa (Thuno.) C.B. Cl.

Helichrysum felinum (Thuno.) Less

Cullunia aculeata (Houtt.) Roesl. var. aculeata

Ficinia trichodes (Schrad.) Benth. \& Hook. \&

Pentaschistis colorata (Steud.) Stapf

Erica cubica l.

IThesium virgatun Lam.

Elegia asperiflora (Nees) kunth

Tetraria ustulata (L, C.B.Cl.

Eheharta ramosa (Thunb.) Thunb.

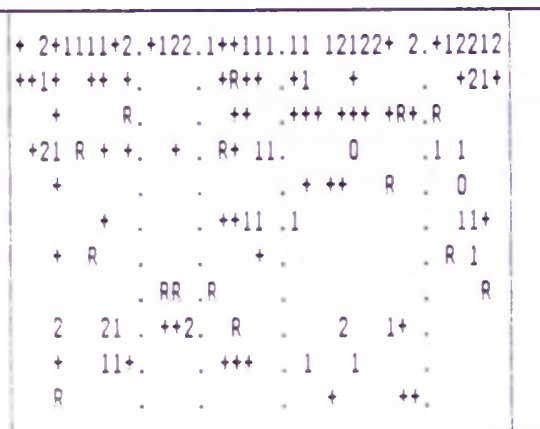

121 
TABLE 1. - Phytosociological table of the Erica hispidula Shrublands on the south slopes of the Bergfontein area, southern Langeberg (continued)

$2223 * 22222222 * 22222222222 * 22222222222222 * 2222222222 * 3333 * 2222222 * 222222222222 * 222222 * 2222$ $9990 * 5566777 * 3335555777 * 223334445577 * 1144444566 * 0000 * 233335 * 11122222466 * 111224 * 6666$ $7890 * 8909346 * 127 ! 267012 * 890890563457 * 2313489534 * 1234 * 734560 * 78902346212 * 456157 * 5678$

Soecies common to comnunities $1.1,1.2 .1,1.2 .2,1.2 .3,1.3 .1,1.3 .2 .1,1.3 .2 .2 .1 .3 .3 \& 1.3 .4$

Stoebe plunosa (L.) Thunt.

clutia alaternoides $L$.

Elegia juncea $i$.

Lodelia neglecta Roen. \& Schult.

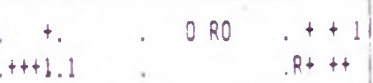

$R+++$

Differential scecies of the Erica hispidula-proted nitida Shrublands (1.4)

Protea nitida Mill.

Theneda triandra forssk.

Forb - bristly leaves (unidentified)

Clutia laxa Eckl. ex Sonc.

Grass fine leaves bulbous base

Calopsis filifornis (Mast.) Linder

Erica deltata Andr.

Tristachya leucothrix Nees

Erica glandulosa Thunb.

Pelargoniu cancicans Spreng.

Protasparagus rubicuncus (Berg.) Oberm

Rhus lucida!. forma lucida

Eragrostis capensis (Thunb.) Trin.

Species common to communities $1.3 .4 \& 1.4$

Diospyros glabra (L.) de Winter

Anthospermum aethiodicum $L$.

Helichrysum cymosun (L.) O. Don subsd. cymosum

montinia caryophyllacea Thunb.

Crassula ericoices Harv.

Rhus rosnarinifolia Vahl.

Eragrostis curvula (Schrad.) Hees

pelargoniun myrehifoliun (L.) L'Herit. var. myrihi.

Species common to communities $1.3 .3,1.3 .4 \& 1.4$

Leucadendion salignum Berg.

Selago dregei Rolfe

Aloe gracilis var. decumbers Reynolds

Species common to communities $1.3 .2 .2,1.3 .3,1.3 .4 \& 1.4$

Phylica inberbis Berg.

Euclea polyandra (L.f.) E. Mey ex Hiern.

Muraltia ciliaris $D C$.

(Leucospermum cuneiforme (8urn. f.) Rourke

Scabiosa columbaria L.

Cymbopogon marginatus (Steud.) Stapf ex Burtt Davy

Hermannia angularis Jaca.

Metalasia Dungens $D$. Don

Senecio crenatus Thunt.

\begin{tabular}{|c|c|c|c|c|c|c|c|c|c|}
\hline . &.+ & . & . & & . & . & . & & $++\ldots+012$ \\
\hline - & . & . & . & 0 & . & . & . & & I $\quad R+,+\phi+\phi$ \\
\hline . & . & . & . & & - & . & . & & t+t+ R. Ot+ \\
\hline . & & . & . & & . & - & . & $R$ & $+1,++$ \\
\hline . & . & . & . & & . & - & . & & I+ . ORR \\
\hline . & . & . & . & & . & . & . & & $+.1++$ \\
\hline . & . & . & . & & . & . & . & & $1 .++$ \\
\hline . & . & . & . & & . & . & . & & .1 \\
\hline
\end{tabular}

Species common to communities $1.2 .2,1.2 .3,1.3 .1,1.3 .2 .1,1.3 .3,1.3 .4 \& 1.4$

Penaea mucronata $L$.

Lanaria lanata (L.) Dur. \& Schinz

Cliffortia siricta mein.

Helichrysum nudifoliun (L.) Less.

Aspalathus opaca Eckl. \& leyh. Subsp. opaca
$1+2212222+1 .++++12.32311$

$0+t 0 \ldots+t+t+$

$++.++\infty$ 
TABLE 1. - Phytosociological table of the Erica hispidula Shrublands on the south slopes of the Bergfontein area, southern Langeberg (continued)

Relevé nunber $\quad 2223 * 2222222 * 2222222222 * 2222222222222 * 2222222222 * 3333 * 2222222 * 22222222222 * 222222 * 2222$ $9990 * 5566777 * 3335555777 * 223334445577 * 1144444566 * 0000 * 233335 * 11122222466 * 111224 * 6666$ $7890 * 8909346 * 1271267012 * 890890563457 * 2313489534 * 1234 * 734560 * 78902346212 * 456157 * 5678$

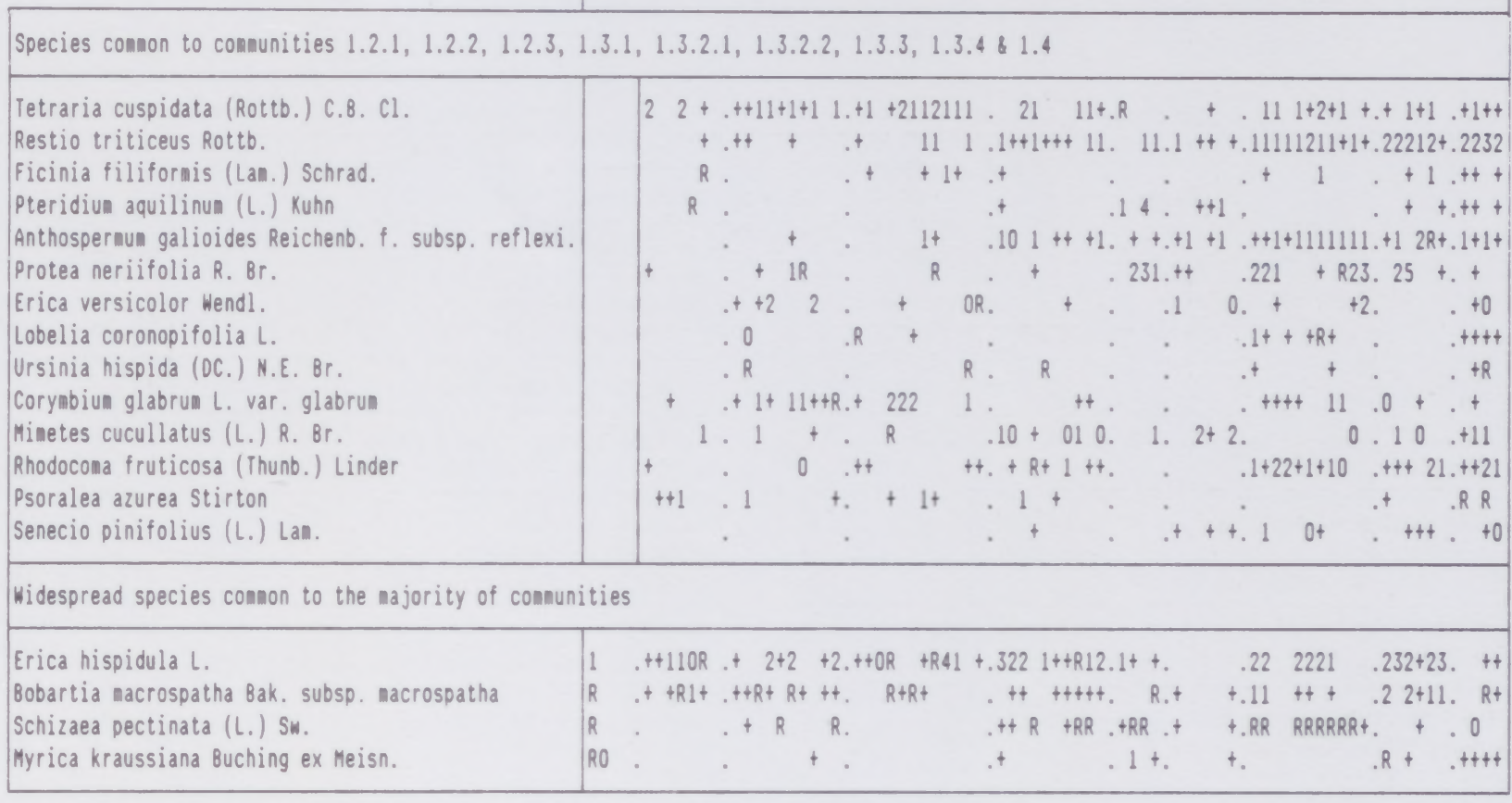

the Langeberg mountain range. A small population of the rare Langeberg endemic species Empleurum fragrans (Rutaceae) was also located close to plot 298 but not in the Erica hispidula-Brunia alopecuroides Shrubland; this represents a range extension of some $30 \mathrm{~km}$ east of its previously recorded range (Williams 1984). Leucadendron radiatum (Proteaceae), also a Langeberg endemic, is found on rocky promontories amongst the more uniform Erica hispidula-Brunia alopecuroides Shrubland.

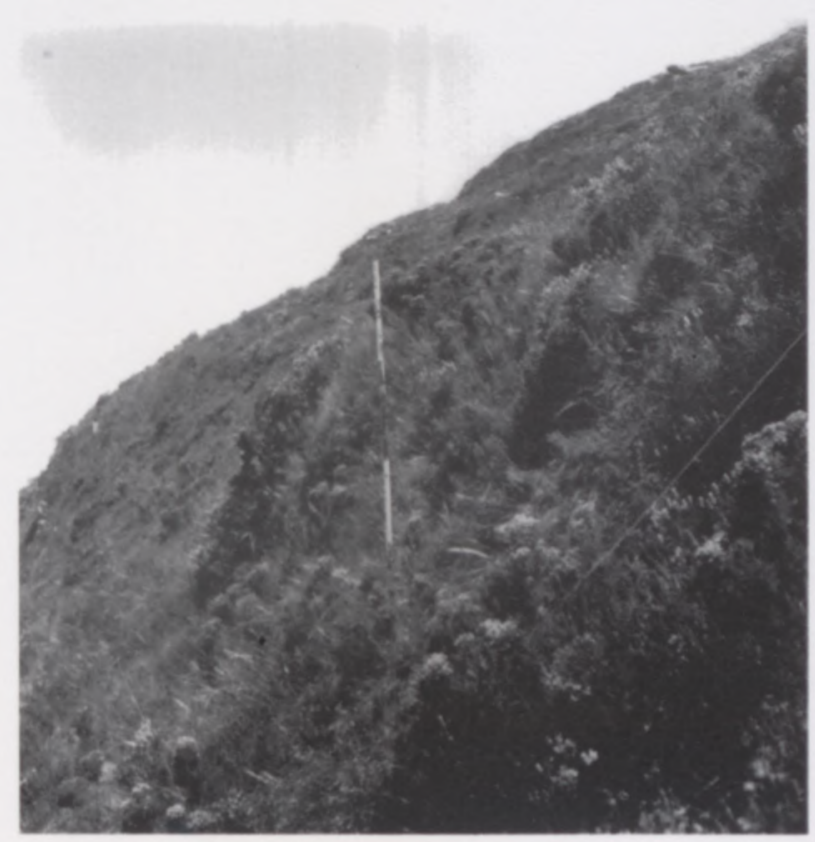

FIGURE 5.-The Erica hispidula-Brunia alopecuroides Shrublands on the high altitude south-facing slopes of Korinteberg.

\subsection{Erica hispidula-Restio inconspicuus Shrublands}

This community, similar to the shrublands of the same name in BWA, comprises most of the shrublands where Restio inconspicuus is present. $R$. inconspicuus is not found in the Erica hispidula-Brunia alopecuroides Shrublands and is sparingly present in the Tetraria bromoidesBerzelia galpinii Shrublands (Table 2). The Erica hispidula-Restio inconspicuus Shrublands described here were sampled on the southern mid- to lower slopes of the Bergfontein area and are characterized by presence of Agapanthus africanus, Berzelia galpinii, Nevillea sp. nov. (Restionaceae) (newly discovered in the Bergfontein area) amongst other species (Table 2).

Penaea cneorum subsp. ovata, Leucadendron spissifolium and Drosera aliceae, all species which prefer moist slopes, occur in the Erica hispidula-Brunia alopecuroides Shrublands and the Erica hispidula-Restio inconspicuus Shrublands.

\subsubsection{Restio inconspicuus-Chondropetalum mucrona- tum Shrublands}

Differential species: Chondropetalum mucronatum, Chironia jasminoides.

Dominant species: Blaeria coccinea, Platycaulos compressus, Restio inconspicuus.

Structural formation: Closed Graminoid Shrubland.

Relationships: Chondropetalum-Restio Tussock Marsh (Boucher 1978); 'Slope-type' Restioid Marsh (Taylor 1978); Erica mollis Fynbos Community (Glyphis et al. 1978); Restio-Hypolaena Subcommunity (Laidler et al. 1978); Restiad Herblands (in part) (Kruger 1979); Sneeu- 
TABLE 2. - A phytosociological table of the Cullumia aculeata var. aculeata Shrublands on the north slopes of Witelsberg (Bergfontein) and Phesantefontein, southern Langeberg

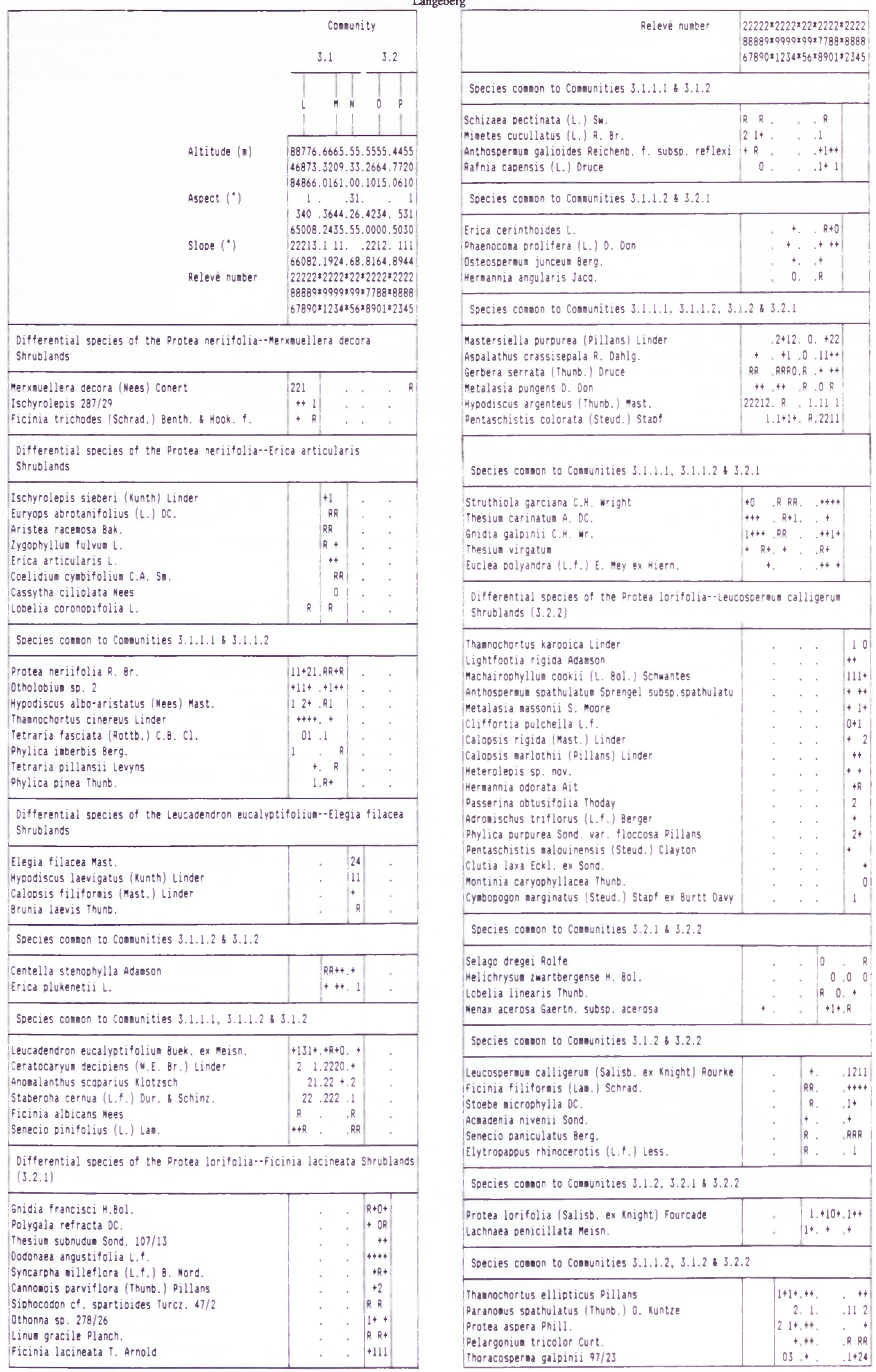


TABLE 2.-A phytosociological table of the Cullumia aculeata var. aculeata Shrublands on the north slopes of Witelsberg (Bergfontein) and Phesantefontein, southern Langeberg (continued)

\begin{tabular}{|c|c|}
\hline Relevé nunber & $\begin{array}{l}22222 * 2222 * 22 * 2222 * 2222 \\
88889 * 9999 * 99 * 7788 * 8888 \\
67890 * 1234 * 56 * 8901 * 2345\end{array}$ \\
\hline \multicolumn{2}{|c|}{ Species comon to comunities $3.1 .1 .1,3.1 .1 .2,3.1 .2+3.2 .2$} \\
\hline $\begin{array}{l}\text { Restio filiforuis Poir. } \\
\text { Erica versicolor Wendl. }\end{array}$ & $\begin{array}{ll}111.1+21.2 \\
1 . R R \quad .0+. & .0 \mathrm{Rt}\end{array}$ \\
\hline \multicolumn{2}{|c|}{ Species connon to Comunities $3.1 .1 .2,3.2 .1 \leqslant 3.2 .2$} \\
\hline $\begin{array}{l}\text { Willdenowia bolusii Pillans } \\
\text { Syncarpha paniculata (L.) B. Nord. } \\
\text { Aspalathus acanthes Eckl. \& Leyh. } \\
\text { Elegia galpinii M.E. Br. } \\
\text { Lightfootia fasciculata (L.f.) A. OC. }\end{array}$ & 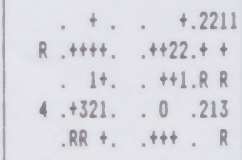 \\
\hline \multicolumn{2}{|c|}{ Species comon to Comunities $3.1 .1 .1,3.1 .1 .2 \leqslant 3.2 .1$} \\
\hline $\begin{array}{l}\text { Osteospernun triquetrun L.f. } \\
\text { Leucospermun cuneiforne (Burn. f.) Rourke } \\
\text { Tetraria cuspidata (Rottb.) C.B. Cl. } \\
\text { Huraltia ciliaris DC. } \\
\text { Bobartia nacrospatha Bak. subsp. nacrospatha } \\
\text { Lanaria lanata (L.) Dur. Schinz. } \\
\text { Metalasia galpinii (L.) Bol. } \\
\text { Tetraria crassa Levyns }\end{array}$ & 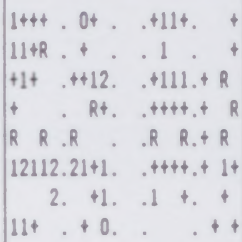 \\
\hline
\end{tabular}

\begin{tabular}{|c|c|}
\hline Relevé nuaber & $\begin{array}{l}22222 * 2222 * 22 * 2222 * 2222 \\
88889 * 9999 * 99 * 7788 * 8888 \\
67890 * 1234 * 56 * 8901 * 2345\end{array}$ \\
\hline \multicolumn{2}{|c|}{ Species comon to Conunities 3.1.1.2, 3.1.2, 3.2.1 $\$ 3.2 .2$} \\
\hline $\begin{array}{l}\text { Protea repens (L.) L. } \\
\text { Hypodiscus striatus (Kunth) Mast. } \\
\text { Oedera inbricata Lan. } \\
\text { Leucadendron salignun Berg. } \\
\text { Rhodocona fruticosa (Thunb.) Linder }\end{array}$ & $\begin{array}{c}R+.31 .2 \quad .011 \\
22.2 \cdot 1+1 .+211 \\
+. R .++R+.++R \\
122+.11 .2222 .1 \\
\text { R+.R.t+. }+11++\end{array}$ \\
\hline \multicolumn{2}{|l|}{ Species comon to Comunities $3.1 .1 .2 \div 3.2 .2$} \\
\hline $\begin{array}{l}\text { Centella virgata (L.f.) Orude var. virgata } \\
\text { Pelargoniun fruticosun (Cav.) willd. } \\
\text { Crassula atropurpurea (Haw.) Dietr. var. atropurpu }\end{array}$ & 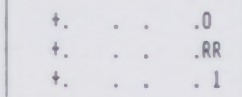 \\
\hline \multicolumn{2}{|l|}{ Widespread species comon to all comunities } \\
\hline $\begin{array}{l}\text { Willdenowia glonerata (Thunb.) Linder } \\
\text { Hypodiscus aristatus (Thunb.) Krauss } \\
\text { Cullunia aculeata (Houtt.) Roesl. var. aculeata } \\
\text { Corynbiun glabru L. var. glabrun } \\
\text { Restio triticeus Rottb. } \\
\text { Tetraria ustulata (L.) C.B. Cl. }\end{array}$ & 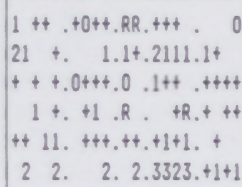 \\
\hline
\end{tabular}

kop Azonal Restioid Fynbos (Campbell 1985); Restio inconspicuus-Anthochortus crinalis Shrublands (McDonald 1993a); Erica hispidula-Anthochortus crinalis Shrublands (McDonald 1993b).

Chondropetalum mucronatum is widely distributed from the eastern Langeberg to Bainskloof and the Cape Peninsula in marshy places (Linder 1985). In the Cape Hangklip area Boucher (1978) records it as occurring on mountain slopes at sites with impeded drainage irrespective of aspect or altitude. This is similarly true on the Langeberg, however, at BWA and MNR (McDonald 1993a \& b), stands of $C$. mucronatum are scattered and simply form part of the vegetation mosaic on south-facing wet slopes. At Bergfontein the distribution of $C$. mucronatum is not so scattered and the species differentiates a distinct community on localized seepages with a 'peaty coarse sand' substratum as it does in the Cape Hangklip mountains (Boucher 1978). Chironia jasminoides is also characteristic of marshy places from the southwestern Cape to the Riversdale District (Bond \& Goldblatt 1984) and at Bergfontein, is almost at the eastern limit of its range.

At Bergfontein the Restio inconspicuus-Chondropetalum mucronatum Shrublands (Figure 6) occur within an altitudinal range from $560 \mathrm{~m}$ to $850 \mathrm{~m}$ on moderate slopes $\left(14^{\circ}-30^{\circ}\right)$ with southeast- to southwest-facing aspects.

This community has an upper stratum dominated by Chondropetalum mucronatum (up to $1.5 \mathrm{~m}$ ) which emerges above a low closed stratum $(<0.5 \mathrm{~m})$ where grasses, restios and ericas are present in more or less equal proportions. Blaeria coccinea (Ericaceae) is the dominant shrub with Platycaulos compressus and Restio inconspicuus (Restionaceae) and Pentameris macrocalycina and Pentaschistis malouinensis (Poaceae) comprising the major part of the graminoid component. The Nevillea sp. nov. recorded here is closely allied to the $N$. obtusissima recorded by Boucher (1978) in the ChondropetalumRestio Tussock Marsh of the Cape Hangklip area.

\subsubsection{Restio inconspicuus-Selago serrata Shrublands}

Differential species: Othonna quinquedentata, Selago serrata, Syncarpha vestita.

Dominant species: Elegia juncea, Leucadendron spissifolium, Pentameris macrocalycina, Pentaschistis malouinensis, Restio inconspicuus, Staberoha cernua.

Structural formation: Low Open to Closed Graminoid Shrubland with sparse emergent shrubs.

Relationships: Tetraria thermalis-Hypodiscus aristatus Community (Kruger 1974); Leptocarpus membranaceus (Calopsis membranacea)-Hypodiscus aristatus Commu-

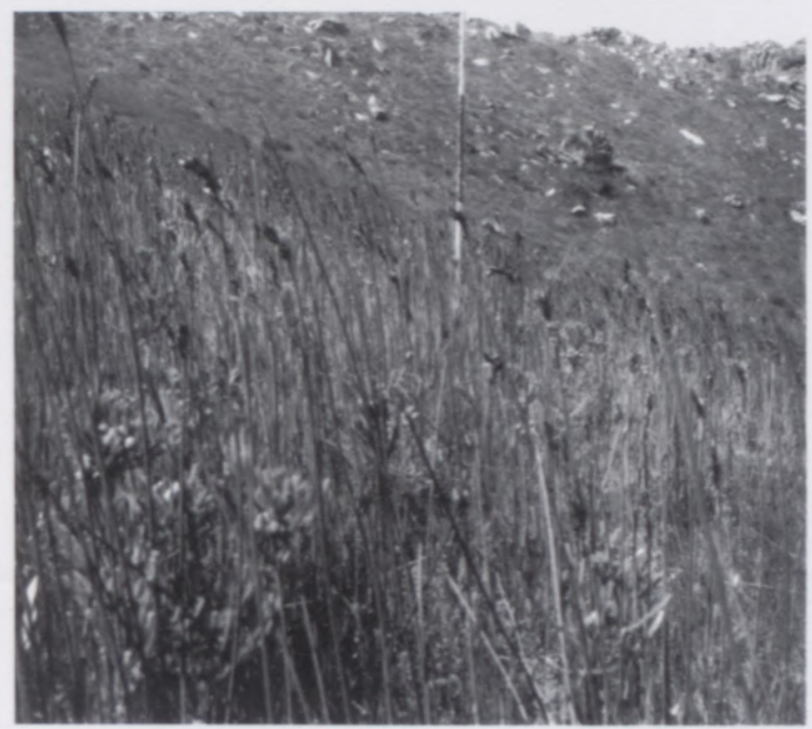

FIGURE 6.-The Restio inconspicuus-Chondropetalum mucronatum Shrublands on southeast- to southwest-facing slopes at Bergfontein. 
nity (McKenzie et al. 1977); Subcommunity C of the Penaea-Erica Fynbos Community (Glyphis et al. 1978); Low Ericoid Open Heath or Open graminoid-heath (Kruger 1979); Erica viridescens-Hypodiscus aristatus Community (Bond 1981); Nuweberg Mesic Ericaceous Fynbos (Campbell 1985); Hypodiscus aristatus-Berzelia intermedia Shrublands (McDonald 1993a).

This community (Figure 7) is found on east- to southwest-facing rocky sites where surface rock was estimated at $32 \%$ on average. Slope inclination ranges from $17^{\circ}-37^{\circ}$ and the sandy Mispah Form soils (lithosols) are well drained. In general this shrubland has a single low stratum $(<1 \mathrm{~m})$ with Psoralea pinnata occasionally emergent to 2 $\mathrm{m}$.

This shrubland community is poorly differentiated. It has only three character species, Othonna quinquedentata, Selago serrata and Syncarpha vestita which are poorly represented. It lacks the moisture-loving species common to communities 1.1 and 1.2.1 but has a strong graminoid component, a characteristic which it shares with the Restio inconspicuus-Chondropetalum mucronatum Shrubland. Apart from the dominant species, Calopsis membranacea and Hypodiscus aristatus (Restionaceae) are well represented, whereas Berzelia galpinii is not prominent. The low cover-abundance of Berzelia galpinii is ascribed to the shallow, rocky, well-drained nature of the soil.

\subsubsection{Restio inconspicuus-Erica melanthera Shrublands}

\section{Differential species: none.}

Dominant species: Berzelia galpinii, Elegia asperiflora, Erica cubica, Pentaschistis malouinensis, Staberoha cernua, Tetraria flexuosa. land.

Structural formation: Low Closed Graminoid Shrub-

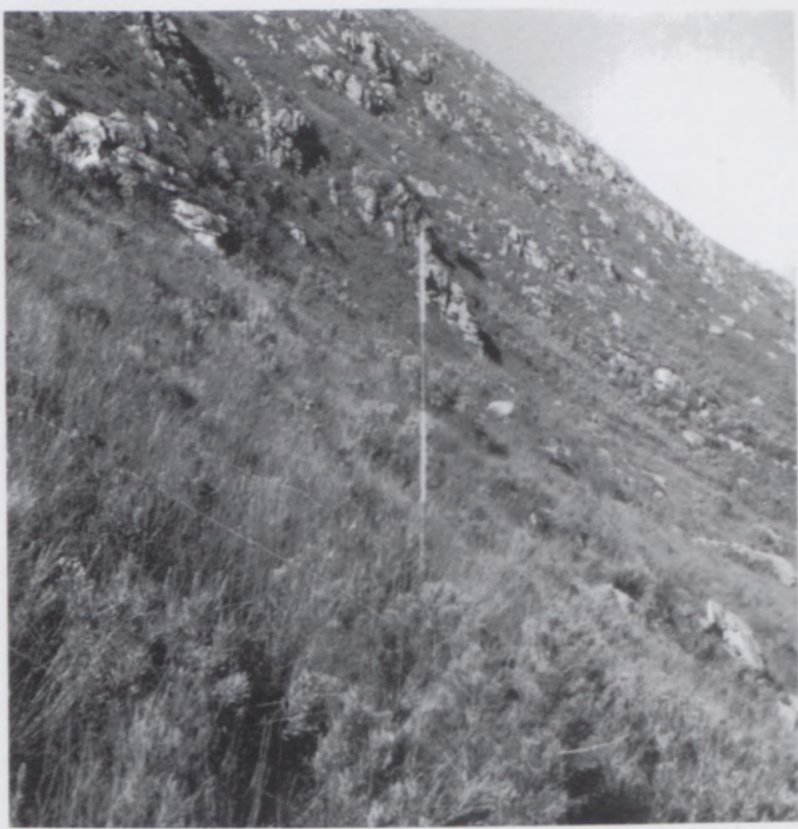

FIGURE 7.-The Restio inconspicuus-Selago serrata Shrublands found on rocky sites with east- to southwest-facing aspects above Bergfontein.

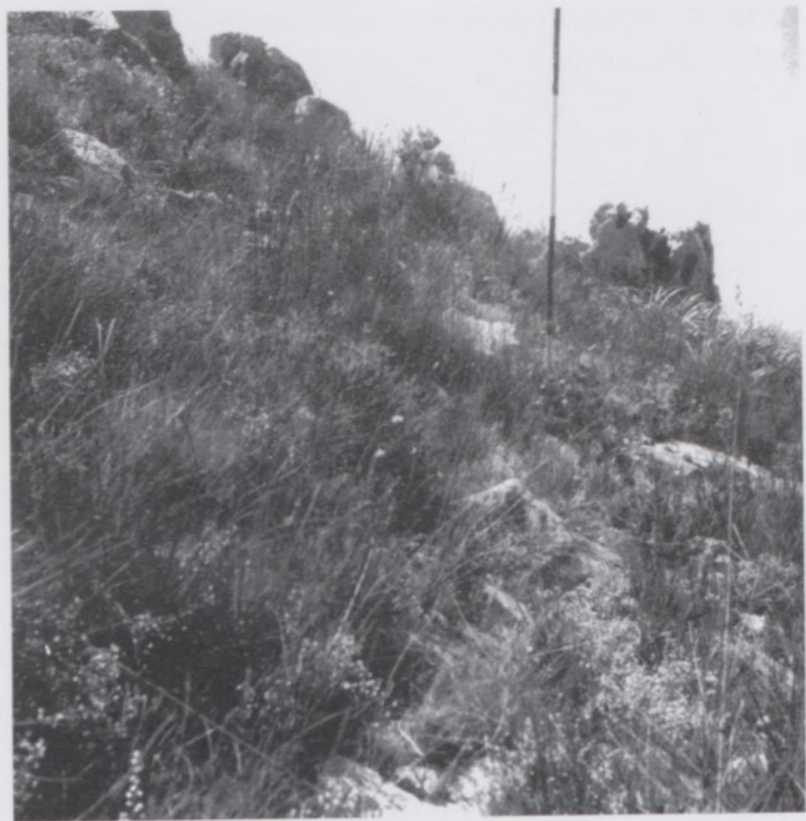

FIGURE 8.-The Restio inconspicuus-Erica melanthera Shrublands on grey shallow sandy lithosols at Bergfontein.

Relationships: as for 1.2.2 above but Berzelia intermedia-Erica melanthera Shrublands (McDonald 1993a) and Leucadendron eucalyptifolium-Erica melanthera Shrublands (McDonald 1993b).

This community (Figure 8 ) is found on a variety of aspects from northeast through south to southwest. The soils are grey sandy shallow lithosols, seldom exceeding $0.3 \mathrm{~m}$ and mostly $<0.2 \mathrm{~m}$ deep. Amounts of exposed rock range from very low to $98 \%$ with most sample sites having $85 \%$ rock cover. Despite this, total vegetation cover is high at $94 \%$.

The absence of differential species in this community is apparently related to the drainage régime. Species such as Berzelia galpinii, Erica cubica and Elegia asperiflora which have a strong preference for sites with impeded drainage, although occurring in other communities, play a dominant role here. Erica melanthera which favours shallow sandy soils is also mainly found in these shrublands, but this species is generally much less common than in the Hypodiscus aristatus-Berzelia intermedia Shrublands of BWA (McDonald 1993a).

The low stature of the community is ascribed to the shallowness of the soil and high percentage rock cover. It could be speculated, however, that some stands would reach mid-high to tall stature in older vegetation.

\subsection{Erica hispidula-Tetraria bromoides Shrublands}

These shrublands include five distinct communities that have $T$. bromoides as the common denominator. $T$. bromoides (Cyperaceae) is a robust leafy sedge that is known for its preference for heavier soils i.e. soils with a high clay fraction, derived from shale or sandstone (Boucher 1978; McDonald 1993a \& b). Of note is the occurrence of Penaea mucronata in these shrublands. This species shows a distinctly different habitat preference (lower, drier slopes) to Penaea cneorum subsp. ovata 
which is found in communities of the Erica hispidulaRestio inconspicuus Shrublands on wetter, higher slopes.

\subsubsection{Tetraria bromoides-Berzelia galpinii Shrublands}

Differential species: none.

Dominant species: Erica hispidula, Leucadendron eucalyptifolium, Penaea mucronata, Tetraria flexuosa.

Structural formation: Low Closed Graminoid Shrubland with Mid-high Emergent Shrubs in some stands.

Relationships: Berzelia-Leucadendron Moist Tall Fynbos (Boucher 1978); Mixed-sclerophyllous Scrub (Kruger 1979); Mangold Wet Proteoid Fynbos (Campbell 1985).

This community (Figure 9) is found on south and southwest-facing slopes at altitudes ranging from $350-550$ $\mathrm{m}$. Soils are derived from sandstone of either the Peninsula Formation or Nardouw Subgroup and are generally 0.5 $\mathrm{m}$ deep, with soils at some sites as shallow as $0.1 \mathrm{~m}$. The average slope of sampled stands is $21.3^{\circ}\left(8^{\circ}-32^{\circ}\right)$. Rockiness is generally low, with an average of $12 \%$, but with some sites having as much as $90 \%$ surface rock. Amount of litter depended on the age of the stand, with the old vegetation having an estimated $75 \%$ litter cover below the shrub canopy.

Two of the stands sampled (relevés $212 \& 213$ ) of the community were located in vegetation estimated to be 16 years or possibly older. The remainder of the samples were in six-year-old vegetation. The two mature stands gave a clear indication of how the Tetraria bromoides-Berzelia galpinii Shrublands would appear over a much wider area when mature. In the mature state they would be classified structurally as Tall Closed Proteoid Shrublands with a Closed Ericoid Shrubland Understorey, in contrast with the structural formation given above.

The Tetraria bromoides-Berzelia galpinii Shrublands are transitional between the Erica hispidula-Restio in-

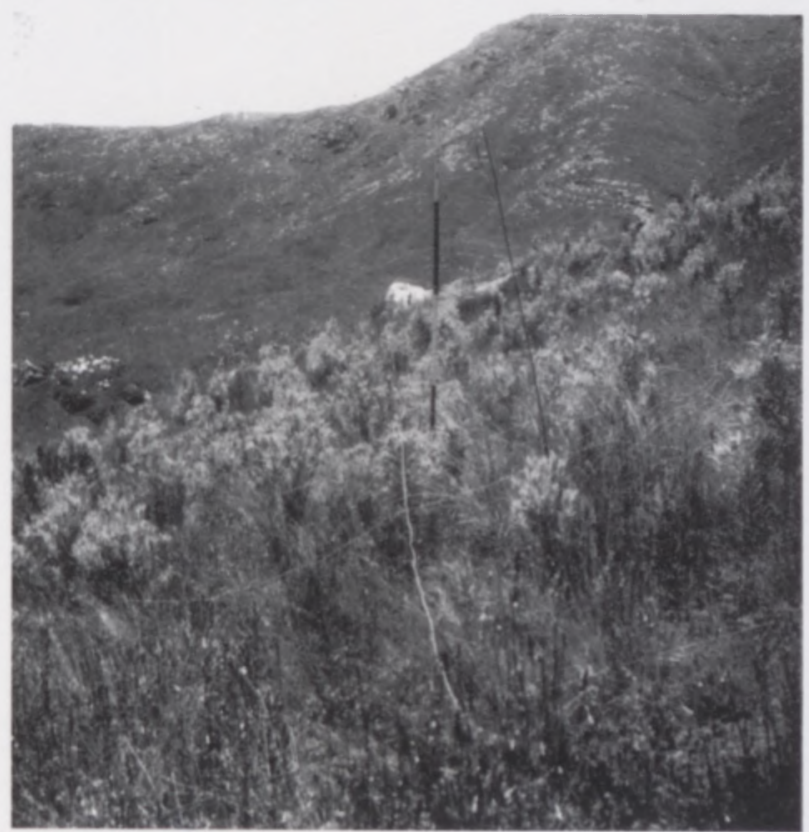

FIGURE 9.-The Tetraria bromoides-Berzelia galpinii Shrublands found on southerly slopes with sandy soils at altitudes from $350-550 \mathrm{~m}$ conspicuus Shrublands on shallow sandy soils and the Erica hispidula-Tetraria bromoides Shrublands on soils with a high clay fraction derived either from shale or an admixture of sandstone and shale. Since the Tetraria bromoides-Berzelia galpinii Shrublands are ecotonal their floristic composition is complex, displaying elements of both the Erica hispidula Shrubland types given above (Table 1).

\subsubsection{Tetraria bromoides-Ischyrolepis hystrix Shrublands}

The two communities found on the Cedarberg Formation shaleband, the Tetraria bromoides-Phylica rubra and Tetraria bromoides-Knowltonia capensis Shrublands have many species in common but also a number of differences (discussed below). However, the absence of many species from these communities which are otherwise generally found in the Erica hispidula-Tetraria bromoides Shrublands sets these shrublands apart. These distinctions are ascribed to soil-related rather than climate-related factors.

\subsubsection{Ischyrolepis hystrix-Phylica rubra Shrublands}

Differential species: Cyclopia sessiliflora, Indigofera sarmentosa, Phylica rubra.

Dominant species: Ischyrolepis hystrix, Leucadendron eucalyptifolium, Phylica rubra, Protea aurea, $P$ neriifolia, Tetraria bromoides.

Structural formation: Tall Proteoid Shrubland with Mid-high, Mid-dense Shrub Understorey and Low Middense Graminoid Field Layer.

Relationships: Broad-sclerophyllous Scrub (Kruger 1979); Protea aurea-Pteridium aquilinum Community (Bond 1981); Outeniqua Wet Proteoid Fynbos (Campbell 1985); Restio inconspicuus-Protea aurea Shrublands (McDonald 1993a); Cliffortia serpyllifolia-Leucadendron eucalyptifolium Shrublands and Widdringtonia nodifloraTetraria bromoides Shrublands (McDonald 1993b).

The Ischyrolepis hystrix-Phylica rubra Shrublands (Figure 10) were sampled on the Cedarberg Formation shaleband, east of Stinkhoutbos, behind Korinteberg. The mean altitude of the sample sites (relevés 301-304), which were located relatively close to each other in mature proteoid fynbos, is $824.5 \mathrm{~m}$. Aspect ranged from southwest to west on shallow $\left(5^{\circ}\right)$ to moderate $\left(22^{\circ}\right)$ slopes. The yellow-brown sandy clay loam soils are classified as Clovelly Form, reaching a mean depth of $0.45 \mathrm{~m}$. Surface rock cover is low, not exceeding $10 \%$ and the soils are moderately to well drained. Litter cover is high $(60-80 \%)$ and the shrub canopy cover is closed (100\%).

The age of the Ischyrolepis hystrix-Phylica rubra Shrublands, estimated at 15 years, could be a factor influencing the difference in species composition between this community and the closely allied but younger Ischymolepis hystrix-Phylica pinea Shrublands found at Bergfontein (see below). Alternatively it may be postulated that geographical separation of the two areas where the shrublands were sampled may account for the differences between them (gamma diversity). These two shrublands may also be interpreted as variants of a broader shaleband community dominated by Cannomois virgata, Erica grata, Ischyrolepis hystrix, Phylica pinea and Protea 


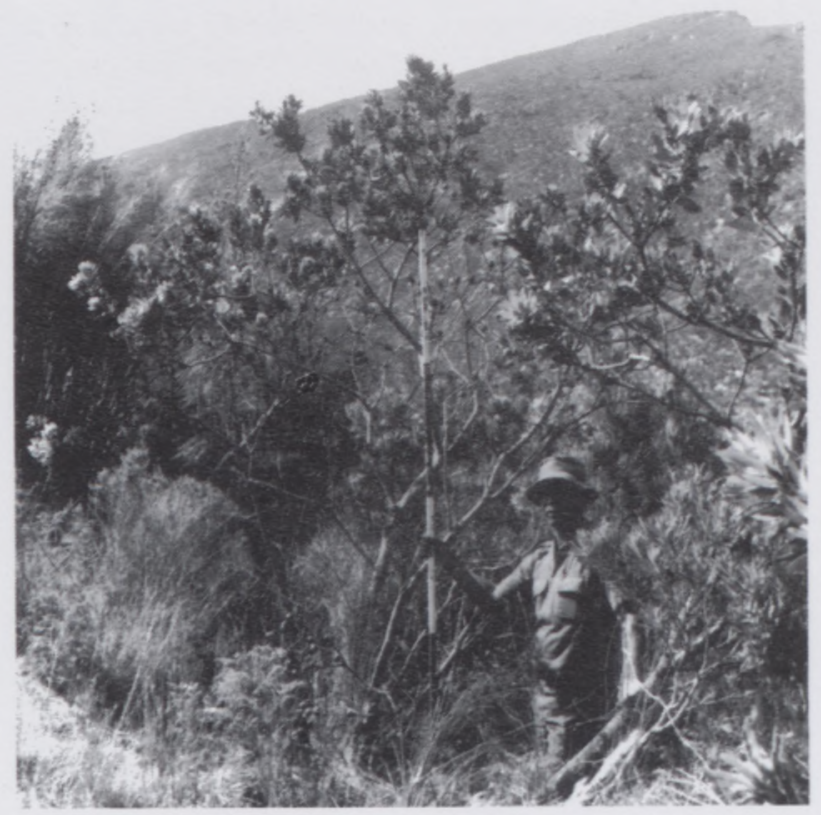

FIGURE 10.-The Ischyrolepis hystrix-Phylica rubra Shrublands found on the Cedarberg Formation shaleband east of Stinkhoutbos. Note the tall Protea aurea shrubs behind the figure.

aurea, since $P$. aurea and Tetraria bromoides dominated shaleband shrublands have been recorded in other parts of the Langeberg (McDonald 1993a \& b). As much as the differential species separate the above two communities from each other, absence of numerous species, the most notable of which are Gnidia galpinii, Lanaria lanata, Penaea mucronata and Struthiola garciana, reinforce the distinction between the two shrublands (Table 2).

\subsubsection{Ischyrolepis hystrix-Phylica pinea Shrublands}

Differential species: Carpacoce spermacocea, Erica coccinea, Knowltonia capensis, Pelargonium cordifolium, Serruria fasciflora.

Dominant species: Cannomois virgata, Ischyrolepis hystrix, Leucadendron eucalyptifolium, Phylica pinea.

Structural formation: Tall Mid-dense to Closed Restioid/Proteoid Shrubland with Sparse Mid-high Shrub Understorey and a Low Mid-dense to Closed Graminoid Shrubland field layer.

Relationships: as for 1.3.2.1 above.

Apart from relevé 227, samples representing the Ischyrolepis hystrix-Phylica pinea Shrublands (Figure 11) were found at altitudes from $300-400 \mathrm{~m}$ on southeast- to southwest-facing slopes of the Cedarberg Formation shaleband, where it traverses the Bergfontein area. Relevé 227 was sampled on a shale lens on a moderate northwest-facing slope above Rooiwaterspruit, where the soil is a $0.3 \mathrm{~m}$ deep, yellow-brown loamy Clovelly Form soil. The soils at Bergfontein were not classified except that they were noted to have a grey-brown orthic A-horizon.

At Rooiwaterspruit, the vegetation in relevé 227 was dominated by Leucadendron eucalyptifolium in the tall (1.5-3.0 m) mid-dense canopy. Non-ericoid shrubs and graminoids combined to form a closed understorey, 1.5 $\mathrm{m}$ high. The community represented by relevé 227 is somewhat different to the Bergfontein Community since many of the typical shaleband-associated species are absent (Table 2). However, it is similar enough to the Ischyrolepis hystrix-Knowltonia capensis Shrublands and different enough from the Ischyrolepis hystrix-Phylica rubra Shrublands for it to be included in the former. The community at Bergfontein was dominated by the tall (1.2$5.0 \mathrm{~m}$ ) Cannomois virgata in the canopy, with a mid-high, mid-dense stratum dominated by Phylica pinea and Protea aurea and a low, closed stratum dominated by restioids.

The Ischyrolepis hystrix-Phylica pinea Shrubland is the only community from which Erica hispidula is totally absent. No explanation for this can be advanced since this species occurs in similar communities in the MNR and BWA (McDonald 1993a \& b). A combination of wetness and shale-derived soil may account for the absence of this species.

\subsubsection{Tetraria bromoides-Hypodiscus striatus Shrublands}

Differential species: Anomalanthus scoparius, Diosma tenella, Heteropogon contortus, Hypodiscus striatus, Peucedanum ferulaceum, Polygala sp.

Dominant species: Anomalanthus scoparius, Erica hispidula, Hypodiscus striatus, Lanaria lanata, Leucadendron eucalyptifolium, L. salignum, Tetraria bromoides.

Structural formation: Low Mid-dense to Closed Graminoid Shrubland with Mid-high to Tall, Sparse to Mid-dense Proteoid Shrubland Overstorey.

Relationships: Mixed-sclerophyllous Scrub (Kruger 1979); Protea neriifolia-Leucadendron eucalyptifoliumErica triceps Community (Bond 1981); Robinson Mesic Proteoid Fynbos (Campbell 1985); Hypodiscus aristatusLeucadendron eucalyptifolium Shrublands (McDonald 1993a); Leucadendron eucalyptifolium-Erica melanthera Shrublands (McDonald 1993b).

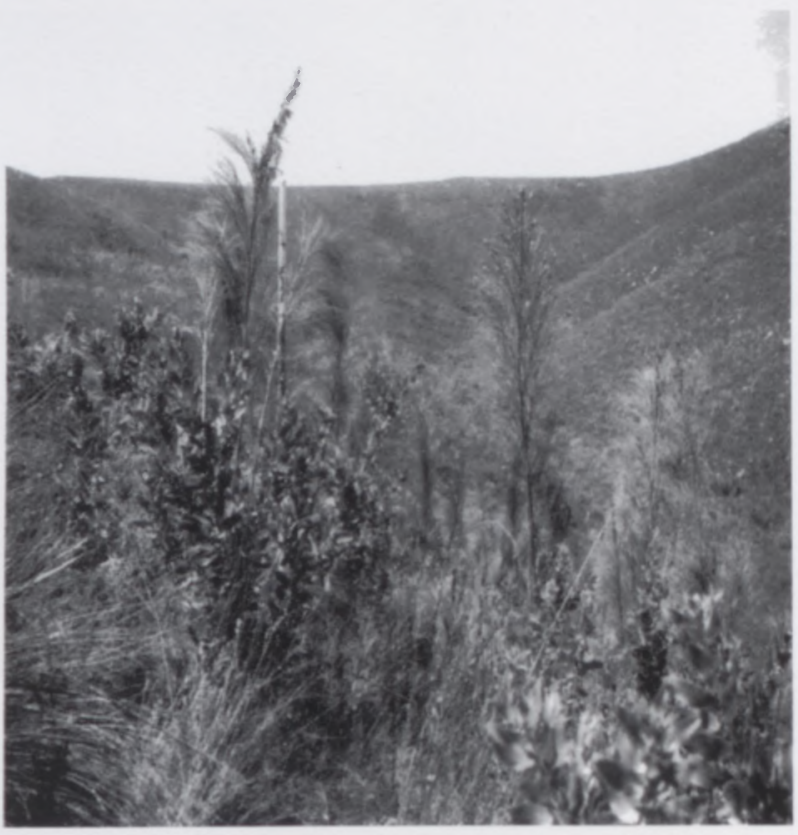

FIGURE 11.-The Ischyrolepis hystrix-Phylica pinea on the Cedarberg Formation shaleband in the Bergfontein area. Note the tall restio, Cannomois virgata, characteristic of this community. 
These shrublands (Figure 12) were sampled on the north and northwest slopes of Koksposberg and on the west-facing mid-slopes of Witelsberg, overlooking Witelsrivier (Bergfontein area). The community occurs at altitudes from $300-550 \mathrm{~m}$ on sites with shallow $(0.2 \mathrm{~m})$ well-drained sandy loam soils, where the slopes are moderate to steep $\left(16^{\circ}-36^{\circ}\right)$ and where rock cover varies from almost nil to $90 \%$.

Presence of Tetraria bromoides points to soils with a higher fertility, however, soil-vegetation relationships of this community are not clear and require further investigation. The community is clearly defined and characterized by differential species (Table 1) which have a preference for stony or rocky well-drained sites. All the differential species are also species of low stature which are found in the understorey of mature stands of the Tetraria bromoides-Hypodiscus striatus Shrublands. Anomalanthus scoparius (Ericaceae) grows as a prostrate dwarf shrub covering stony soil but not over rocks (E.G.H. Oliver pers. comm.) and the erect Hypodiscus striatus (Restionaceae) is found as tussocks in shallow pockets of soil. Heteropogon contortus is a subtropical $\mathrm{C}_{4}$ grass which Bond (1981) recorded as differential for his Protea nitida Community (Waboomveld) on fertile soils. At Bergfontein, $H$. contortus is faithful to the Tetraria bromoides-Hypodiscus aristatus Shrublands and not found at all in the Erica hispidula-Protea nitida Shrublands described below. Diosma tenella (Rutaceae) is a shrublet which Williams (1982) described as 'rather rare' but having a wide tolerance for different soil types, from shales to sandy gravels and silcrete.

From Table 1 it is seen that there are numerous species which, although not differential or dominant, are strongly represented in this community: Acmadenia trigona, Leucadendron salignum, Lanaria lanata, Penaea mucronata, Staberoha cernua, Tetraria flexuosa and Rhodocoma fruticosa.

One or two strata were found in these shrublands at the time of sampling. One stratum was found where the shrubs were co-dominant with the graminoid component up to a height of $1 \mathrm{~m}$. Two strata occurred where the shrubs, mainly proteoids such as Leucadendron eucalyptifolium and Protea neriifolia, exceeded $1 \mathrm{~m}$, forming a sparse to mid-dense overstorey. The immaturity of the vegetation gave a false impression of the potential structure of the community, which is predicted to become a tall closed proteoid shrubland with a low closed ericoid or restioid shrubland understorey when mature (as in relevés 261 \& 262).

\subsubsection{Tetraria bromoides-Protea coronata Shrublands}

Differential species: Alepidea capensis, Erica $\mathrm{cf}$. zwartbergense, Protea coronata.

Dominant species: Erica hispidula, Lanaria lanata, Leucadendron eucalyptifolium, Tetraria bromoides.

Structural formation: Mid-high Closed Proteoid Shrubland with Low Closed Graminoid Shrubland Understorey or a Low Closed Graminoid Shrubland with Sparse emergent Proteoid shrubs.
Relationships: Broad-sclerophyllous Scrub (Kruger 1979); Outeniqua Wet Proteoid Fynbos (Campbell 1985); Wet Proteoid Fynbos (Rebelo et al. 1991).

This community (Figure 13) occurs on the lower southeast-, southwest- and west-facing slopes of Koksposberg in the Bergfontein area. At plot 221 the soil is derived from Cedarberg Formation shale, whereas at the remainder of the plots (214-216 \& 247) the soils are apparently derived from accumulated material at the footslopes. The soils are well-drained shallow $(0.2-0.3 \mathrm{~m})$ fine-textured grey-brown to dark brown sandy loams, with negligible surface rock cover.

The distribution of Protea coronata is strongly related to edaphic factors with this species favouring heavy soils with a high clay fraction (Rourke 1980). At Bergfontein, Protea coronata occurs on sandy loam soils but, contrary to Rourke's description where Protea coronata is said to form 'densely massed stands', the species occurs as scattered individuals. Frequent fires may have excluded $P$. coronata, a reseeding proteoid, at three of the five sample sites. Alternatively these sites may be marginally more mesic and $P$ coronata, which prefers wetter sites (Rourke 1980), may have been outcompeted by Protea neriifolia and Leucadendron eucalyptifolium (Table 2). Even though the Tetraria bromoides-Protea coronata Shrubland is poorly characterized by the differential species Alepidea capensis, Erica cf. zwartbergense and $P$. coronata, it is substantiated by absence of numerous species found in the Tetraria bromoides-Hypodiscus striatus Shrublands on the one hand and the Tetraria bromoides-Protea nitida Shrublands on the other. It also has many species in common with the latter community, in particular the ubiquitous 'waboomveld' species Montinia caryophyllacea and Rhus rosmarinifolia. Helichrysum cymosum is not a true differential species of the Tetraria bromoides-Protea coronata Community as stated by Rebelo et al. (1991) since this species is also well represented in the more mesic Erica

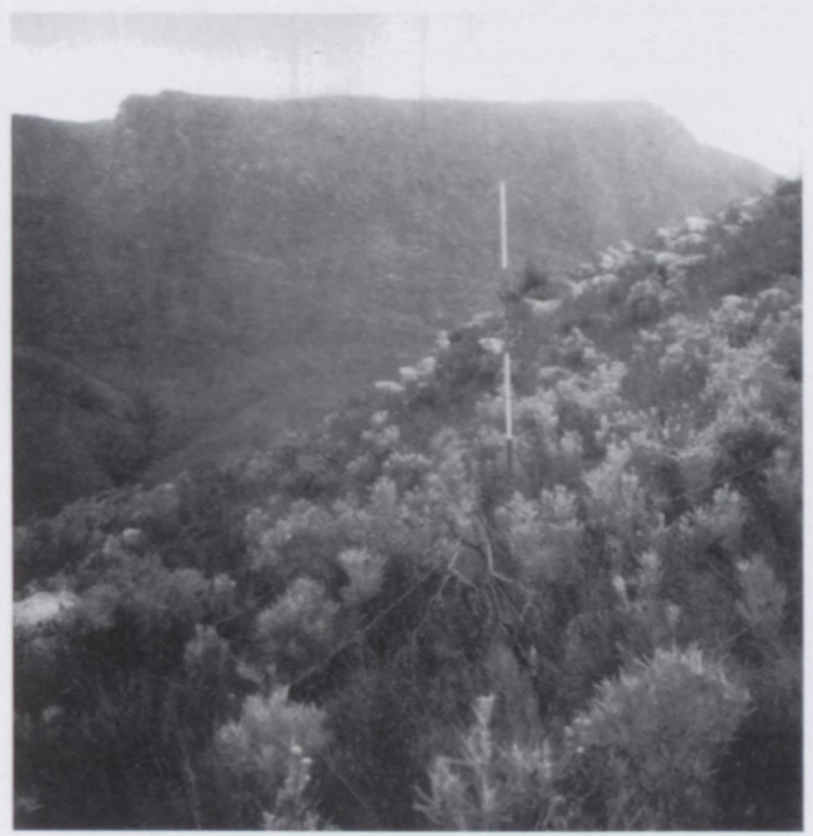

FIGURE 12.-The Tetraria bromoides-Hypodiscus striatus Shrublands on the west-facing slopes of Koksposberg with the south slopes of Witelsberg visible behind. 


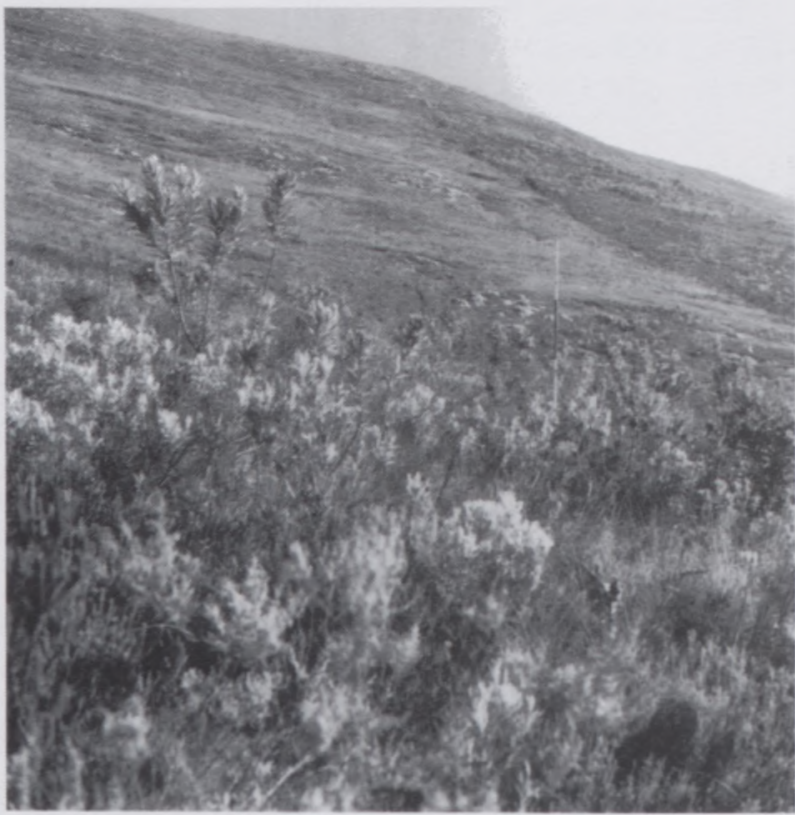

FIGURE 13.-The Tetraria bromoides-Protea coronata Shrublands on the lower slopes of Koksposberg, in the Bergfontein area.

hispidula-Protea nitida Shrublands which were not sampled or described by these authors.

Two strata were found in these shrublands. Graminoids are marginally more abundant than woody shrubs in the low stratum, which was the dominant stratum when sampled. In relevé 247 , the low stratum had been disturbed by grazing animals. The upper stratum was dominated by mid-high proteoid shrubs, Leucadendron eucalyptifolium and Protea neriifolia. Following Campbell's (1985) system, the Tetraria bromoides-Protea coronata Shrublands would be placed in the Mesic Proteoid Subseries, however, based on floristic composition and relationships, the community is placed unequivocally in Wet Proteoid Fynbos.

Aloe gracilis Haw. var. decumbens Reynolds was found in this community and the Erica hispidula-Protea nitida Shrublands. Reynolds (1950) gives the distribution of this endemic variety of Aloe gracilis as from near Garcia's Pass westwards to Kleinberg at altitudes 272-364 m. Records of A. gracilis var. decumbens at Bergfontein represents a range extension for this species on the Langeberg, $30 \mathrm{~km}$ east of Garcia's Pass. Future searches could reveal that it may occur further east, perhaps beyond the Gouritz River.

\subsection{Erica hispidula-Protea nitida Shrublands}

Differential species: Anthospermum aethiopicum, Calopsis filiformis, Clutia laxa, Eragrostis capensis, Erica glandulosa, E. peltata, Pelargonium candicans, Protasparagus rubicundus, Protea nitida, Themeda triandra.

Dominant species: Lanaria lanata, Leucadendron salignum, Protea nitida, Restio triticeus, Themeda triandra.

Structural formation: Closed Graminoid Shrubland with Mid-high Sparse to Open Proteoid Overstorey.

Relationships: 'Waboomveld' (Taylor 1963, 1978; Taylor \& Van der Meulen 1981); Protea arborea ( $P$ nitida)
Pseudo-Savannah (Taylor 1969); Protea arborea-Rhus angustifolia Community (Werger et al. 1972); ProteaTetraria Dry Short Fynbos (Boucher 1978); Restio gaudichaudianus (Ischyrolepis gaudichaudiana)-Lobostemon glaucophyllus Community (McKenzie et al. 1977); Protea arborea $(P$. nitida) Tall Broad-sclerophyllous Shrubland or Open Shrubland with Heathland (Kruger 1979); Protea nitida Community (Waboomveld) [Outeniqua Mountains] \& Protea nitida-Protea repens Community [Swartberg] (Bond 1981); Protea nitida Woodland ('Waboomveld') (Taylor 1984); Rooiberg Talus Asteraceous Fynbos (Campbell 1985); Ischyrolepis gaudichaudiana-Myrsine africana High Closed Shrubland (McDonald 1983, 1988).

Protea nitida is most often found on colluvial soils on debris or talus slopes but may also be found on fine-textured soils on lower mountain slopes (Taylor 1978; Kruger 1979; R.M. Cowling pers. comm.). These soils generally have a higher nutrient status than that of leached sandstone soils, due to colluvial mixing of soil derived from various parent materials such as sandstone, granite or shale. Fynbos with $P$. nitida as a prominent, if not dominant shrub or tree, is found throughout the Fynbos Biome and according to Taylor (1978) and Kruger (1979) characterizes a distinct formation, 'waboomveld'. The term 'waboomveld' encompasses more than one community where $P$ nitida is present, but throughout the distribution range of this species from the northwestern to the southern Cape there is a remarkably constant group of associated species including Anthospermum aethiopicum, Ischyrolepis gaudichaudiana, Montinia caryophyllacea, Rhus rosmarinifolia and Themeda triandra. Campbell (1985) explicitly stated that $P$ nitida is diagnostic and must be present for Mountain Fynbos to be classified as Talus Asteraceous Fynbos but he also indicated that $P$. nitida occurs in other communities as well.

At Bergfontein these shrublands (Figure 14) have either two or three strata. $P$. nitida forms a sparse canopy up to $2 \mathrm{~m}$ high. Where present the second stratum from $0.5-1.2 \mathrm{~m}$ is dominated by Leucadendron salignum and the low stratum $0.0-0.5 \mathrm{~m}$ by Themeda triandra. The community was restricted to well-drained east-facing slopes above Witelsrivier, directly opposite the west-facing slopes of Koksposberg where the closely related Tetraria bromoides-Protea coronata Shrublands occur. The altitude at which these shrublands are found ranges from $300-350 \mathrm{~m}$ with the slopes averaging $19^{\circ}$. Annual precipitation is estimated at $600-700 \mathrm{~mm}$ (Dent et al. 1987). The soils with $\mathrm{pH} 4.9(\mathrm{n}=3)$ are a shallow $(0.2 \mathrm{~m})$ welldrained mix of sandstone debris from the Peninsula Formation sandstone and Cedarberg Formation shale. They are classified as Oakleaf Form soils. Rock cover ranges from $10-40 \%$.

Apparently the Erica hispidula-Protea nitida Shrublands at Bergfontein are not burnt often, so the composition of the understorey is not attributed to regular fires or other disturbances. The grassy understorey is rather attributed to soil factors since the grasses in the community are $\mathrm{C}_{4}$ grasses which show preference for more fertile soils (Bond 1981; Cowling 1983a \& b; Linder 1989). This community qualifies for inclusion in Campbell's (1985) Rooiberg Talus Asteraceous Fynbos on the basis of presence of Protea nitida and $20 \%$ grass cover. However, 
the lack of asteraceous 'elytropappoid' species and the high grass cover $(70 \%)$ indicate closer affinities to Grassy Fynbos (sensu Cowling \& Holmes 1992).

A stand of tall $P$. nitida with an understorey composed almost entirely of grasses was located on deep sandy soils on the plateau below the upper north slopes of Witelsberg. This community was not sampled but was observed to be quite different in structure and composition from the Erica hispidula-Protea nitida Shrublands. Further investigation, not possible in this study due to the fire, would be required to clarify the relationships.

\section{Erica versicolor-Agathosma ovata Shrublands}

Differential species: Adromischus triflorus, Agathosma ovata, Centella virgata var. congesta, Crassula atropurpurea var. atropurpurea, C. muscosa, C. rupestris, Crassula sp. (unidentified), Erica tenuis, Ficinia elongata, Lampranthus deltoides, Lobelia pubescens var. pubescens, Senecio aizoides.

Dominant species: Agathosma ovata, Ehrharta ramosa, Erica melanthera, E. versicolor, P.hylica purpurea var. floccosa.

Structural formation: Sparse to Open Low Graminoid Shrubland.

\section{Relationships: unclear.}

The estimated annual precipitation for Koksposberg is 500-600 mm (Dent et al. 1977). However, the north-facing slopes are in a rain shadow as well as being equatorially oriented. They are therefore hot and dry and probably receive much less rainfall than the above estimate. A similar situation also exists on the upper north-facing slopes of Witelsberg. These dry, rocky slopes (Figure 15) with high incoming radiation host the Erica versicolorAgathosma ovata Shrublands. The soils are lithosols (Mispah Form), are shallow and acid (pH 3.4 in $1 \mathrm{~mol} / \mathrm{h}$ $\mathrm{CaCl}_{2}$ ) and consist merely of accumulated sand and organic material in shallow depressions amongst the rocks.

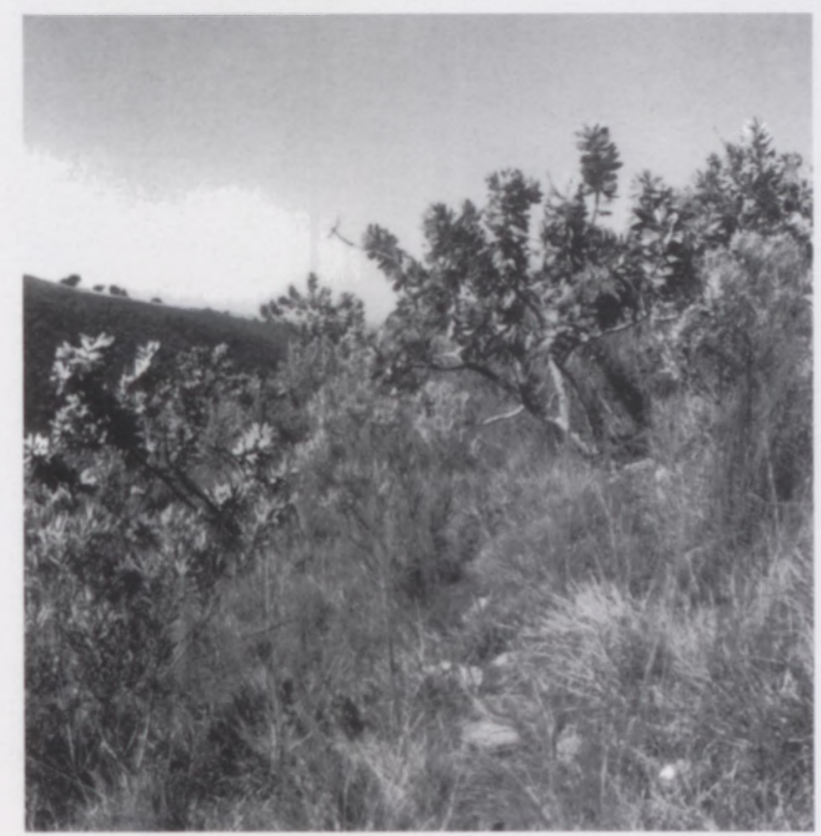

FIGURE 14.-The Erica hispidula-Protea nitida Shrublands on the east-facing slopes, west of Bergfontein, above Witelsrivier.

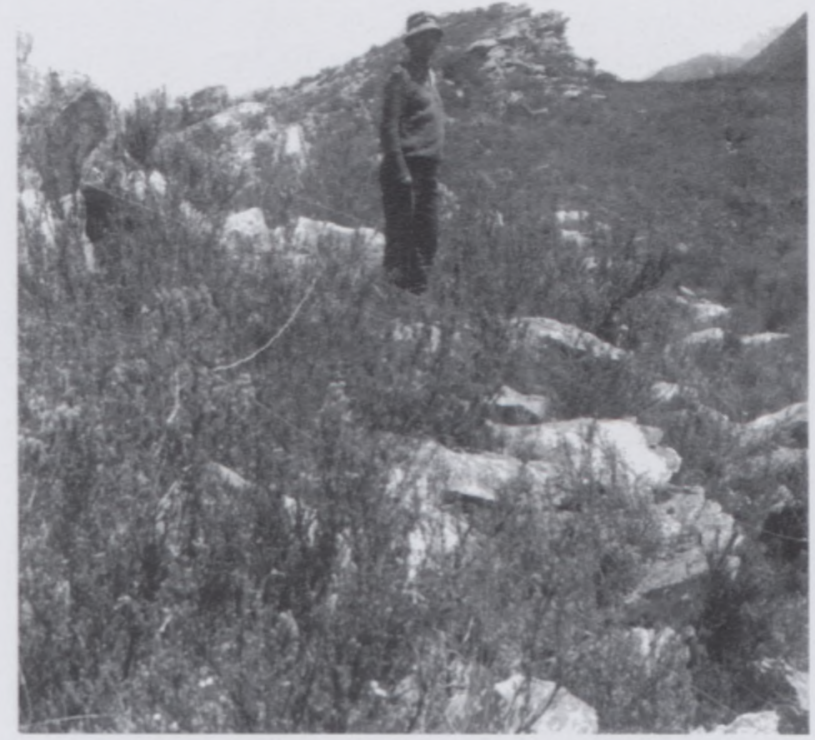

FIGURE 15.-The Erica versicolor-Agathosma ovata Shrublands found on the rocky north slopes of Koksposberg and Witelsberg.

The parent rock of Koksposberg is Peninsula Formation sandstone and rock cover is $90 \%$ in the four relevés (208211) representing this community. On Koksposberg the community is found at altitudes from $550-606 \mathrm{~m}$ on slopes varying from almost flat $\left(7^{\circ}\right)$ on the ridge to moderately steep $\left(18^{\circ}\right)$ on the north face. On Witelsberg, consisting of Nardouw Subgroup sandstone, the community is found from $788 \mathrm{~m}$ to $1090 \mathrm{~m}$.

Two strata are present in the Erica versicolor-Agathosma ovata Shrublands. The upper stratum is from 0.5 $1.2 \mathrm{~m}$ and is dominated by the shrubby Erica versicolor. The lower stratum where shrubs and graminoids are codominant is from $0-0.5 \mathrm{~m}$. Dominant species in this stratum are Ehrharta ramosa and Erica melanthera. However, the prevalence of succulent species in the genera $A d$ romischus, Aloe, Crassula, Lampranthus and Senecio which prefer dry, rocky sites gives this community its character and sets it apart from all other communities described for the Bergfontein area (relevés not included in Table 1 or Table 2). A. ovata also favours a dry, rocky habitat as does Oldenburgia paradoxa which grows in rock crevices forming large, compact, rounded masses of short shoots.

A detrended correspondence analysis (DCA) (Hill $1979 \mathrm{~b})$ of the Bergfontein data showed a clear distinction between the Erica versicolor-Agathosma ovata Shrubland and the other shrublands. DCA Axis I represented a moisture gradient and the Erica versicolor-Agathosma ovata Shrublands are towards the drier end of the gradient. DCA Axis II apparently represented a soil depth/rockiness gradient, showing that the community inhabits the rockiest sites with shallowest soils in the study area.

\section{Cullumia aculeata var. aculeata Shrublands}

The Cullumia aculeata Shrublands include the nonErica hispidula shrublands represented by Table 2. All these shrublands are on the northern slopes of the 
Langeberg range adjacent to the Little Karoo. Most of the samples were taken in the Phesantefontein area; the exceptions are relevés 278-281 which were recorded on the north slopes of Witelsberg before the May 1991 fire.

\subsection{Cullumia aculeata-Leucadendron eucalyptifolium Shrublands}

This community is found on the mid- to upper slopes immediately east of Aasvoëlkrans. They are mesic proteoid shrublands and are separated into three distinct communities with Leucadendron eucalyptifolium as the common factor.

\subsubsection{Leucadendron eucalyptifolium-Protea neriifolia Shrublands}

Two communities are found in the Leucadendron $\mathrm{eu}$ calyptifolium-Protea neriifolia Shrublands. P. neriifolia links these shrublands, setting them apart from the Leucadendron eucalyptifolium-Elegia filacea Shrublands where $P$. neriifolia is absent. These shrublands have affinity to the Tetraria bromoides-Hypodiscus striatus Shrublands found on the southern slopes at Bergfontein with Anomalanthus scoparius and Hypodiscus striatus, differential species of the latter community, being found here as well.

\subsubsection{Protea neriifolia-Merxmuellera decora Shrub- lands}

Differential species: Ficinia trichodes, Ischyrolepis sp., Merxmuellera decora.

Dominant species: Hypodiscus argenteus, Lanaria lanata, Merxmuellera decora.

Structural formation: Mid-high Closed Graminoid Shrubland.

Relationships: Proteoid Zone Fynbos (Taylor 1978); Broad sclerophyllous- Scrub or Open Scrub (Kruger 1979); Sanddrift Mesic Proteoid Fynbos (Campbell 1985); affinities with Tetraria bromoides-Hypodiscus striatus Shrublands and Tetraria bromoides-Protea coronata Shrublands described above.

This community (Figure 16) is represented by five relevés $(286-290)$ at altitudes from $630-850 \mathrm{~m}$ on the northeast- to northwest-facing slopes east of Aasvoëlkrans. These slopes are moderate, with shallow $(0.2 \mathrm{~m})$, yellow-brown sandy loam soil, classified as Glenrosa Form. The parent material of the soil is taken to be Nardouw Subgroup sandstone, but the heavy nature of the soil (relevés 286-289) suggests a high clay fraction possibly derived from a clay lens or local exposure of the Cedarberg Formation shale. This requires verification but the vegetation reflects a soil with a relatively higher nutrient status (see below). Surface rock cover is low to moderate (3-40\%) except in relevé 290 which is different from the other samples. It was located on a rocky sandstone outcrop with $95 \%$ rock cover. The soil is of the Mispah Form, but the floristic composition of the relevé places it in the Protea neriifolia-Merxmuellera decora Shrublands.
At the time of sampling, the vegetation was seven years old. Depending on the site, the community had one or two strata, with height not exceeding $1.2 \mathrm{~m}$. The shrubland was thus classified as above, a Mid-high Closed Graminoid Shrubland, but it is predicted that with time the community would mature to a Tall Closed Proteoid Shrubland with a Graminoid Understorey.

The Protea neriifolia-Merxmuellera decora Shrublands are poorly differentiated from the closely allied P.rotea neriifolia-Erica articularis Shrublands based on the differential species of the first community. Merxmuellera decora presents problems with identification in mature vegetation since it usually flowers only in the immediate post-fire phase; Ischyrolepis sp. (Table 2) was not identified and Ficinia trichodes was poorly represented. Absence of the differential species of the latter community from the Protea neriifolia-Merxmuellera decora Shrublands provides a sounder basis for the separation of the two communities.

\subsubsection{Protea neriifolia-Erica articularis Shrublands}

Differential species: Aristea racemosa, Cassytha ciliolata, Coelidium cymbifolium, Erica articularis, Euryops abrotanifolius, Ischyrolepis sieberi, Zygophyllum fulvum.

Dominant species: Ceratocaryum decipiens, Elegia galpinii, Leucadendron salignum, Staberoha cernua.

Structural formation: Low Closed Herbland or Low Mid-dense to Closed Graminoid Shrubland.

Relationships: Proteoid Zone Fynbos (Taylor 1978); Broad-sclerophyllous Scrub or Open Scrub (Kruger 1979); Elegia galpinii-Metalasia pulcherrima Community (Outeniqua Mountains) and Protea repens-Protea lorifolia-Hypodiscus striatus Community (Swartberg) (Bond 1981); Mesic Mountain Fynbos (Moll et al. 1984); Thomas Dry Proteoid Fynbos (Campbell 1985). Leucadendron eucalyptifolium-Staberoha cernua Shrublands (McDonald 1993a).

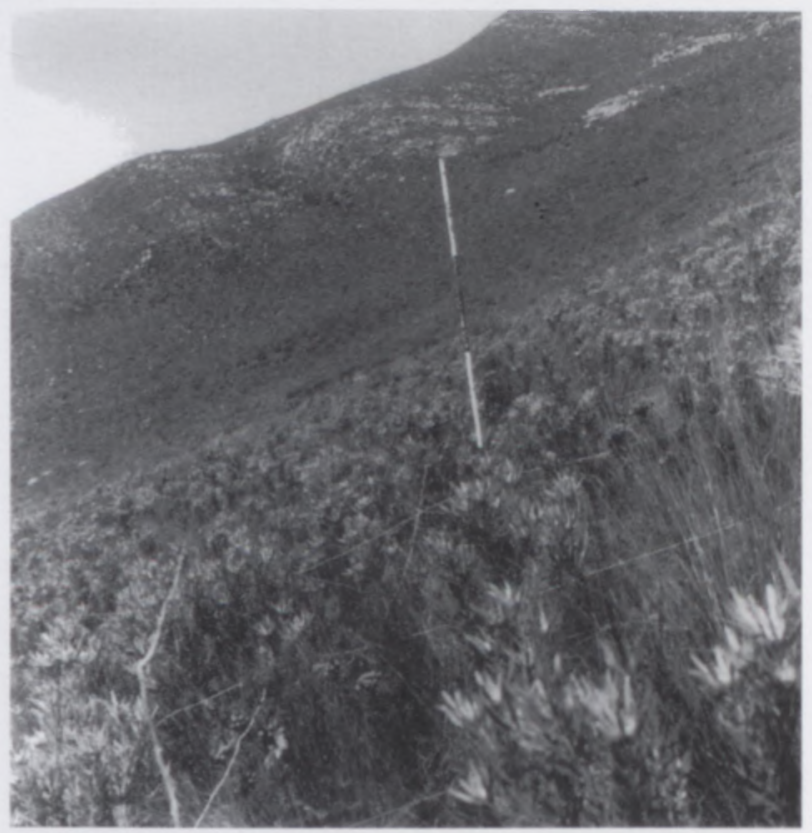

FIGURE 16.-The Protea neriifolia-Merxmuellera decora Shrublands found on the northeast- to northwest-facing slopes east of Aasvoëlkrans, above Phesantefontein. 
This community (Figure 17) occurs on the same northfacing slopes as the Protea neriifolia-Merxmuellera decora Shrublands but at lower altitudes (590-630 m). The shallow $(0.2-0.3 \mathrm{~m})$, light grey-brown soils were classified as Glenrosa Form (relevés 291-293) and Mispah Form (relevé 294). All the relevés were situated on shallow slopes $\left(9^{\circ}-14^{\circ}\right)$, between rock outcrops, where rock cover was very low except in relevé 294 where it was estimated at $20 \%$

The differential species of the Protea neriifolia-Erica articularis Shrublands are not constant throughout the community, nor do they have high cover-abundance. Of these species, Erica articularis and Ischyrolepis sieberi have highest cover-abundance whereas the remaining species are rare (Table 2). The very rare legume, Coelidium cymbifolium, previously known from only two collections, Muir's type collection and a collection of Middlemost (Granby 1980), was found in relevés 293 and 294 and appears to be narrowly endemic to this community. Protea aspera, a prostrate rhizomatous shrublet, was also recorded in this community and the Leucadendron eucalyptifolium-Elegia filacea Shrublands (see below and Table 2). This species is best known from Onrust to Bredasdorp but has also been recorded from an isolated population in the Langkloof, east of Garcia's Pass (Rourke 1980). Records of Protea aspera at Phesantefontein represent an extension of the range of the Langkloof population $5 \mathrm{~km}$ west of Garcia's Pass.

The Protea neriifolia-Erica articularis Shrublands are dominated by restios such as Ceratocaryum decipiens, Elegia galpinii, Mastersiella purpurea, Restio filiformis, Thamnochortus ellipticus and Staberoha cernua, all species preferring mesic to dry habitats. T. ellipticus was originally collected by Muir in the Phesantefontein area and is only known from this, the type locality (Linder 1985).

\subsubsection{Leucadendron eucalyptifolium-Elegia filacea Shrublands}

Differential species: Calopsis filiformis, Elegia filacea, Hypodiscus laevigatus.

Dominant species: Elegia filacea, Protea repens.

Structural formation: Low Closed Graminoid (Restioid/Cyperoid) Shrubland with Tall Open Proteoid Overstorey.

Relationships: as for 3.1.1.2 above and Leucadendron eucalyptifolium-Protea lorifolia Shrublands (McDonald 1993a).

Two relevés, 295 and 296, represent this community (Figure 18). It is found below the Leucadendron eucalyptifolium-Erica articularis Shrublands, and is floristically transitional between them and the $C$. aculeataProtea lorifolia Shrublands. Both relevés were at $530 \mathrm{~m}$ on slopes with a shallow gradient. The soil which is 0.25 $0.3 \mathrm{~m}$ deep is a light grey sandy loam with quartz pebbles and gravel on the surface. Rock cover is nil and the soils are classified as Glenrosa Form.

Elegia filacea is most often found on accumulated sand and the sandy nature of the soil accounts for its presence here. In this community it dominates the understorey in

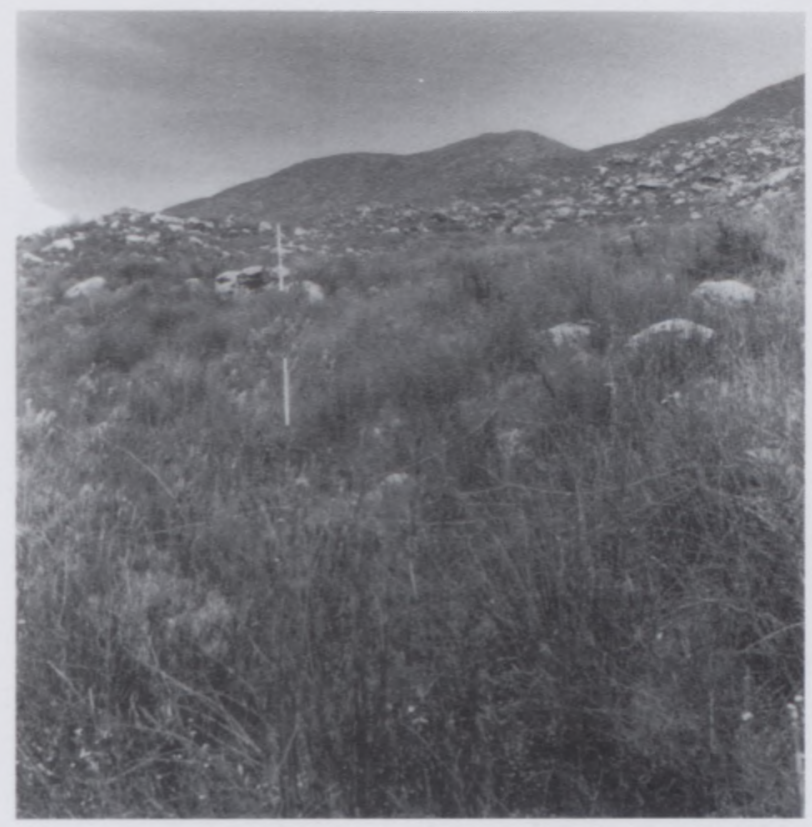

FIGURE 17.-The Protea neriifolia-Erica articularis Shrublands on the lower north-facing slopes above Phesantefontein.

which grasses are absent and sedges few, mainly of the genus Ficinia. Low shrubs play a subordinate role in the understorey composition, but the presence of Protea aspera, which also favours sandy soil, should be noted. The upper (tall) stratum is composed of proteoid shrubs with Protea repens dominant.

The rare, endemic Erica rhodantha Guth. \& Bol. was found in this community. This species is apparently restricted to the fynbos shrublands of the lower north slopes of the Langeberg in the region of Garcia's Pass. Few collections of this species have been made (E.G.H. Oliver pers. comm.).

The Leucadendron eucalyptifolium-Elegia filacea Shrublands are the same as the Leucadendron eucalyptifolium-Protea lorifolia Shrublands described from BWA (McDonald 1993a), but for the purposes of this paper are treated as slightly different. The difference is based on the presence of Calopsis filiformis, Hypodiscus laevigatus, Protea aspera and Thamnochortus ellipticus at Phesantefontein and not at BWA

\subsection{Cullumia aculeata-Protea lorifolia Shrublands}

This community, found at the driest extreme of the south-north climatic gradient of the Langeberg, is divided into two: the Protea lorifolia-Ficinia laciniata Shrublands (which fall within the Leucadendron eucalyptifolium-Protea lorifolia Shrublands of the BWA) and the Protea lorifolia-Leucospermum calligerum Shrublands (which are equivalent to the Passerina obtusifolia-Leucospermum calligerum Shrublands at BWA). The Cannomois parviflora-Passerina obtusifolia Shrublands found at BWA (McDonald 1993a) were not identified as a separate community at Phesantefontein but as part of the Passerina obtusifolia-Leucospermum calligerum Shrublands. 


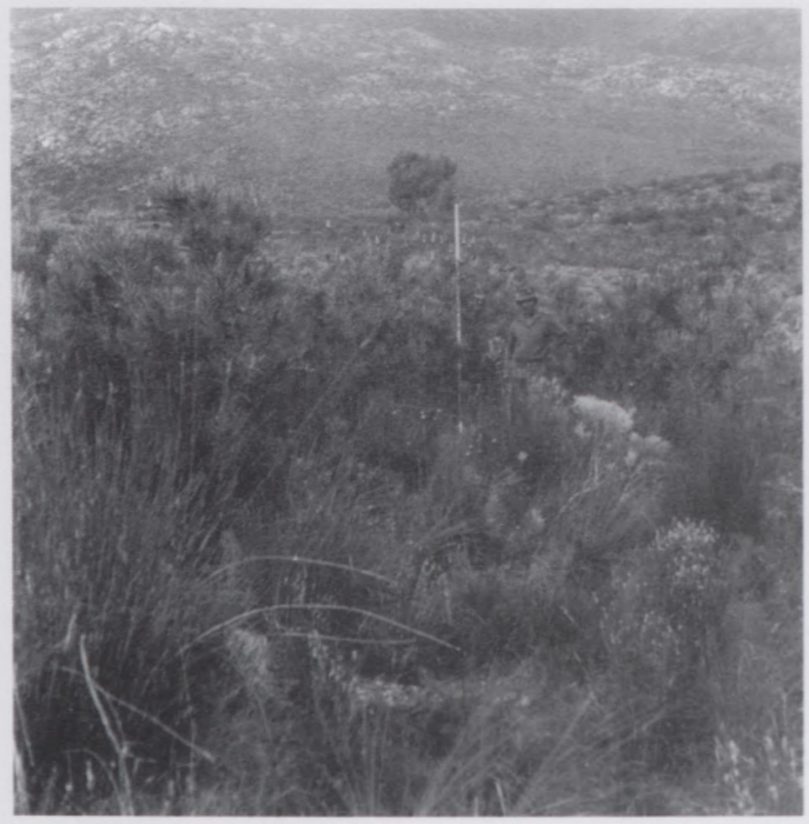

FIGURE 18.-The Leucadendron eucalyptifolium-Elegia filacea Shrublands.

\subsubsection{Protea lorifolia-Ficinia laciniata Shrublands}

Differential species: Cannomois parviflora, Dodonaea angustifolia, Ficinia laciniata, Gnidia francisci, Linum gracile, Othonna sp., Polygala réfracta, Siphocodon cf. spartioides, Syncarpha milleflora, Thesium subnudum.

Dominant species: Leucadendron salignum, Pentaschistis colorata, Hypodiscus aristatus, Tetraria ustulata.

Structural formation: Low Mid-dense Sedgeland or Graminoid Shrubland with Mid-high Sparse Proteoid Shrubs in some stands.

Relationships: Proteoid Zone Fynbos (Taylor 1978); Broad-sclerophyllous Scrub or Open Scrub (Kruger 1979); Elegia galpinii-Metalasia pulcherrima Community (Outeniqua Mountains) and Protea repens-Protea lorifolia-Hypodiscus striatus Community (Swartberg) (Bond 1981); Mesic Mountain Fynbos (Moll et al. 1984); Thomas Dry Proteoid Fynbos (Campbell 1985); Lel'cadendron eucalyptifolium-Protea lorifolia Shrublands (McDonald 1993a).

This community was not found or sampled at Phesantefontein. The four relevés (278-281) representing the Protea lorifolia-Ficinia laciniata Shrublands were situated on the moderately steep, rocky, north-facing slopes of Witelsberg on the Bergfontein transect at altitudes from $545-730 \mathrm{~m}$. The soils are shallow $(0.1-0.15 \mathrm{~m})$ sandy Mispah Form lithosols and surface exposure of rock was estimated to be $91 \%$ on average.

This community is well differentiated from the other shrublands represented in Table 2 . This could be ascribed either to geographical separation of these relevés from the Phesantefontein area or to the habitat where this shrubland occurs not being present or not sampled at Phesantefontein. Topographically, in rockiness and in climatic regime, the north-facing slopes of Witelsberg closely resemble the north-facing slopes of Deception Ridge in BWA (McDonald 1993a). At Phesantefontein, however, moderately steep, rocky, well-drained slopes of the same type are absent. The 'habitat explanation' for the absence of this community at Phesantefontein therefore seems the most plausible.

Leucadendron salignum, Hypodiscus aristatus, Pentaschistis colorata and Tetraria ustulata dominate the community at this early stage of its development ( 6 years old), forming a low, mid-dense graminoid shrubland. As these shrublands mature it is predicted that the proteoid shrubs, Protea lorifolia and Protea repens will form a mid-high to tall, open to mid-dense overstorey.

\subsubsection{Protea lorifolia-Leucospermum calligerum Shrublands}

Differential species: Adromischus triflorus, Anthospermum spathulatum, Calopsis rigida, C. marlothii, Cliffortia pulchella, Clutia laxa, Cymbopogon marginatus, Hermannia odorata, Heterolepis sp. nov., Lightfootia rigida, Machairophyllum cookii, Metalasia massonii, Montinia caryophyllacea, Passerina obtusifolia, Pentaschistis malouinensis, Phylica purpurea, Thamnochortus karooica.

Dominant species: Elegia galpinii, Leucospermum calligerum, Thoracosperma galpinii, Willdenowia bolusii.

Structural formation: Low to Mid-high Mid-dense Graminoid Shrubland with Sparse emergent Proteoid Shrubs in some stands.

Relationships: Arid Fynbos (Taylor 1978; Kruger 1979); Phylica axillaris-Felicia filifolia Community (Outeniqua Mountains) and Passerina obtusifolia-Felicia filifolia-Pentaschistis eriostoma Community (Swartberg) (Bond 1981); Dry Mountain Fynbos (Moll et al. 1984); Sebrafontein Dry Asteraceous Fynbos (Campbell 1985); Dry Proteoid Fynbos (Rebelo et al. 1991); Passerina obtusifolia-Leucospermum calligerum Shrublands (McDonald 1993a); Leucadendron eucalyptifolium-Hypodiscus argenteus Shrublands in part (McDonald 1993b).

This community (Figure 19) is found on the low, arid northern foothills of Phesantefontein at altitudes from $450-520 \mathrm{~m}$. The slope varies from almost level $\left(5^{\circ}\right)$ to $14^{\circ}$ and the soils are mostly shallow $(<0.1 \mathrm{~m})$, welldrained, rocky (75-98\%) Mispah Form soils. The exception is the Glenrosa Form soil of relevé 285 which is 0.25 $m$ deep with an estimated surface rock cover of only $3 \%$ ! Relevé 283 was situated on a rocky outcrop and was the only site where Passerina obtusifolia was recorded. The silcrete-capped mesas at Phesantefontein present a similar substratum and habitat to that found in relevé 283 and therefore, although these mesas were not sampled, it is predicted that they will support shrublands of the Protea lorifolia-Leucospermum calligerum type (Phesantefontein) or the Cannomois parviflora-Passerina obtusifolia type (Witbooisrivier; McDonald 1993a).

These shrublands are well defined with numerous differential species. Heterolepis sp. nov. (as yet undescribed), the succulent Machairophyllum cookii (Mesembryanthemaceae) and Thamnochortus karooica are endemic to this community. Species with single occurrences have been retained in Table 2 specifically for this community, for the sake of completeness. They are, however, not truly differential. 
Leucospermum saxatile which is endemic to the Arid Fynbos east of Garcia's Pass (Rourke 1972) was noted in this community on the northern footslopes of Witelsberg prior to the May 1991 fire. Serruria balanocephala Rourke ined. found in this community at Witbooisrivier (McDonald 1993a) has not been found at Phesantefontein nor further east. Leucospermum erubescens recorded from Springfontein immediately west and at Garcia's Pass directly east of Phesantefontein was also not encountered at the latter locality and is apparently also not found much further east than Garcia's Pass.

Owing to the extensive fires on the Langeberg east of Garcia's Pass in the recent past, much of the vegetation on the lower northern slopes of the range is immature and thus not suitable for sampling. However, it can be confidently stated that the community named the Passerina obtusifolia-Leucospermum calligerum Shrublands (McDonald 1993a) and the Protea lorifolia-Leucospermum calligerum Shrublands (this paper) extend along the base of the Langeberg from Barrydale to the Gouritz River, fringing the Little Karoo.

\section{DISCUSSION AND CONCLUSIONS}

The hierarchical classification and description of the fynbos communities of the Bergfontein and Rooiwaterspruit-Phesantefontein areas satisfies the primary objective of the study. It also provides a framework which, together with differential species for each community, is a starting point for describing the communities over a wider area on the southeastern Langeberg. Further sampling in other areas would serve to test this classification and would most likely identify additional communities.

From the perspective of management of the fynbos of the Langeberg mountain catchments, this classification is perhaps too detailed and, as may be argued, too floristically orientated. However, it is important to be able to

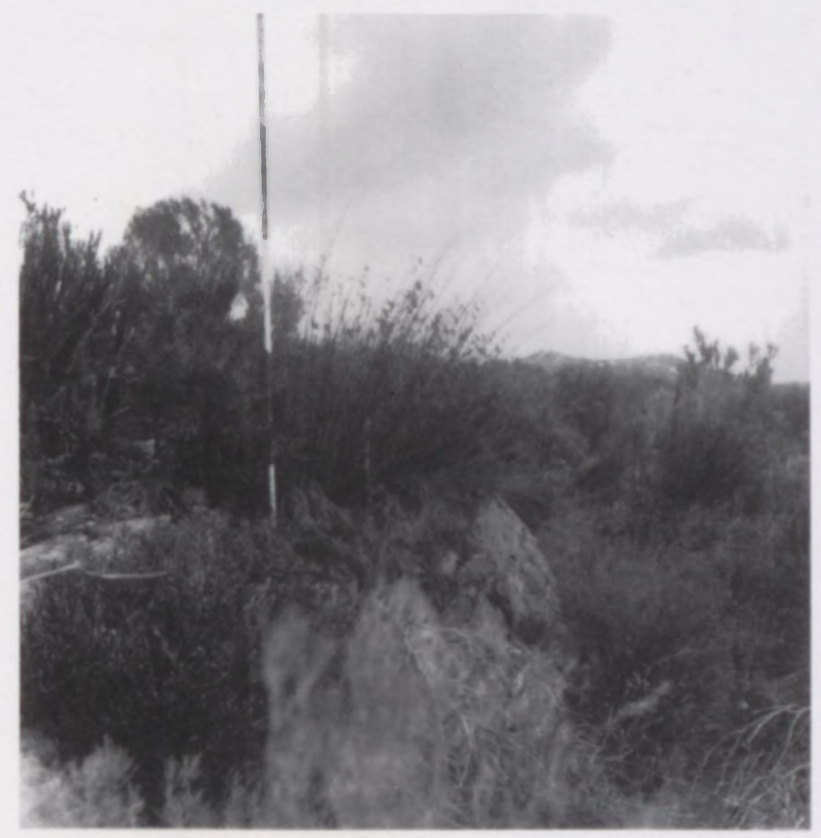

FIGURE 19.-The Protea lorifolia-Leucospermum calligerum Shrublands found on the flow, arid foothills of the Phesantefontein area. classify plant communities at all stages of the succession. This has raised much debate (Cowling \& Holmes 1992) and it has been generally concluded that when applying structural classifications it is only possible to satisfactorily classify mature fynbos plant communities (Campbell 1985). Finding stands of mature fynbos ( $>10$ years old) is becoming increasingly difficult (Campbell 1985; this study) so applying structural classifications becomes equally difficult. Despite the complexity of the vegetation in this study, the successful hierarchical classification of the mainly immature fynbos, and the determination of character species for communities highlights a major advantage in using the floristic classification approach. If suitably interpreted, by using the character species for different communities, the complex taxonomy of the fynbos need not be the reputed daunting obstacle for managers of fynbos vegetation (Bond 1981).

In general, the communities described for the Bergfontein and Rooiwaterspruit-Phesantefontein areas are similar to those described by McDonald (1993a \& b) for the Boosmansbos Wilderness Area and the Marloth Nature Reserve. Degree of difference or conversely, similarity of the fynbos communities of the southern Langeberg, which is beyond the scope of this paper, can now be assessed. A proposed synthesis is now possible which is aimed at being generally applicable in the Langeberg fynbos shrublands, and hopefully beneficial to the conservation and management of these shrublands.

Apart from the purely descriptive reference to environmental conditions pertaining to each community, little indication is given of environmental variables as the causal agents of species distribution and species composition of communities. This forms a separate aspect to the study of the Langeberg vegetation and will be reported separately (McDonald unpublished data).

The Erica versicolor-Agathosma ovata Shrubland found on Koksposberg does not fit well within the hierarchy of communities. This was evident in both the phytosociological tables and the ordination of the data. Many of the fynbos species found generally on the south slopes of the Bergfontein area are found in this community but the succulent differential species indicate an azonal xeric community. A clear definition of this community and its relationships to other fynbos vegetation will only be possible given additional samples. It is predicted to occur on the sandstone outliers of the Langeberg east of Garcia's Pass as described and possibly on the Aasvogelberg near Albertinia.

Garcia's Pass has historically given ready access to the Muiskraal area at its northern entrance. It has thus been an area much visited by botanists since the time of Thunberg and Masson in the late 1700's. Many collections of Langeberg north slope endemic species have Muiskraal as the type locality, notably many of Muir's collections. Many of these species are considered rare. Some of these species such as Coelidium cymbifolium and Erica thodantha have been found in this survey at Phesantefontein which lies just west of Muiskraal. The intensity of sampling in this study has, however, been too limited to cover the area adequately. It is proposed therefore that the areas on the north slopes of the Langeberg west and east of 
Garcia's Pass require thorough botanical investigation. If these areas have high numbers of endemic plant species as is indicated by this survey, these north slopes of the Langeberg deserve a higher conservation status than is currently the case.

\section{ACKNOWLEDGEMENTS}

The hospitality of the Oosthuizen family of the Farm Bergfontein is gratefully acknowledged. Mr C. Martens, Forester at Grootvadersbosch, gave invaluable support; he and Messrs C. Ruiters and J. Solomon, also of Grootvadersbosch, who assisted with strenuous fieldwork, are thanked sincerely. The advice and encouragement of Prof. R.M. Cowling and Dr C. Boucher at times when it was most needed is much appreciated. My wife and family and my colleagues at the Stellenbosch Herbarium. National Botanical Institute, are thanked for their support during this project. Two anonymous referees are also thanked for their suggested improvements to the manuscript.

\section{REFERENCES}

BOND. P. \& GOLDBLATT. P. 1984. Plants of the Cape Flora: a descriptive catalogue. Journal of South African Botany Supplementary Vol. 13.

BOND, W.J. 1981. Vegetation gradients in southem Cape Mountains. M.Sc, thesis, University of Cape Town.

BOND, W.J., VLOK. J. \& VIVIERS, M. 1984. Variation in seedling recruitment of Cape Proteaceae after fire. Joumal of Ecology 72 : 209-221.

BOUCHER. C. 1978. Cape Hangklip area. II. The vegetation. Bothalia 12: $455-497$

BOUCHER. C. 1987. A phytosociological studv of transects through the western Cape coastal foreland. Ph.D. thesis, University of Stellenbosch.

CAMPBELL. B.M. 1985. A classification of the mountain vegetation of the Fynbos Biome. Memoirs of the Botanical Suney of South Africa No. 50.

CAM'L ELL. B.M.. COWLING, R.M.. BOND, W.. KRUGER. F.J. \& MLABORATORS 1981. Structural characterization of vegei'n in the Fynbos Biome. South African National Scientitic rammes Report No. 52. CSIR. Pretoria.

COW] R.M. \& GXABA. T. 1990. Effects of a fynbos overstorey b on understorey community structure: implications for the intenance of community-wide species richness. South African Joumal of Ecology 1: 1-7.

COWLING. R.M. cá HOLMES. P.M. 1992. Flora and vegetation. In R.M Cowling. The ecology of funbos: nutrients, fire and diversity Oxford University Press, Cape Town.

COWLING. R.M. 1983a. The occurrence of $\mathrm{C}_{3}$ and $\mathrm{C}_{4}$ grasses in fynbos and allied shrublands in the southeastern Cape. South Africa. Oecologia 58: 121-127.

COWLING. R.M. 1983b. Phytochorology and vegetation history in the southeastern Cape. South Africa. Journal of Biogengraphy 10: $393-419$.

COWLING, R.M. 1984. A syntaxonomic and synecological study in the Humansdorp region of the Fynbos Biome. Bothalia 14: 283-298

DENT, M.C., LYNCH. S.D. \& SCHULZE. R.E. 1987. Mapping mean annual and other rainfall statistics over southern Africa. Water Research Commission Report No. 27

ESLER. K.J. \& COWLING. R.M. 1990. Effects of density on the reproductive output of Protea lepidecarpodendron. South African Joumal of Botany 55: 29-33.

FUGGLE. R.F. 1981. Macro-climutic pattems within the Funbos Biome. National Programme for Environmental Sciences, Final Report. December 1981

FUGGLE. R.F. \& ASHTON, E.R. 1979. Climate. In J. Day, W.R. Sieg fried. G.N. Louw \& M.L. Jarman. Fynbos ecology: a preliminan sinthesis. South African National Scientific Repor No. 40.

GLYPHIS. J.. MOLL, E.J. \& CAMPBELL. B.M. 1978. Phytosociological studies on Table Mountain. South Africa: 1. The Back Table Joumal of South African Botany 44: 281-289.

GR.ANBY. R. 1980. Revision of the genus Corelidium (LiparieateFabaceae). Opera Botanica 54: $1-47$.
HILL, M.O. 1979a. TWINSPAN-a FORTRAN program for arranging multivariate data in an ordered two-way table by classification of the individuals and attributes. Section of Ecology and Systematics, Cornell University, Ithaca, New York.

HILL. M.O. 1979b. DECORANA-a FORTRAN program for detrended correspondence analysis and reciprocal averaging. Section of Ecology and Systematics, Cornell University, Ithaca, New York.

KRUGER. F.J. 1979. South African heathlands. In R.L. Specht, Heathlands and related shrublands. Ecosistems of the World, Vol. 9A. Elsevier, Amsterdam.

LAIDLER, D., MOLL, EJ., CAMPBELL, B.M. \& GLYPHIS, J. 1978. Phytosociological studies on Table Mountain, South Africa: 2. The Front Table. Joumal of South African Botany 44: 291-295.

LINDER. H.P. 1985. Conspectus of the African species of Restionaceae. Bothalia 15: 387-503.

LINDER. H.P. 1989. Grasses in the Cape Floristic Region: phytogeographical implications. South African Journal of Science 85: 502505 .

MCDONALD, D.J. 1983. The vegetation of Swartboschkloof, Jonkershoek, Cape Province. South Africa. M.Sc. thesis, University of Cape Town.

MCDONALD. D.J. 1988. A synopsis of the plant communities of Swartboschkloof, Jonkershoek. Cape Province. Bothalia 18: 233260)

MCDONALD, D.J. 1993a. The vegetation of the southern Langeberg. Cape Province. 1. The plant communities of the Boosmansbos Wilderness Area. Bothalia 23: 129-151.

MCDONALD DJ.1993b. The vegetation of the southern Langeberg. Cape Province. 2. The plant communities of the Marloth Nature Reserve. Bothalia 23: 153-174.

MCKENZIE. B. 1978. A quantitative and qualitarive study of the indigenous forests of the southwestern Cape. M.Sc. thesis, University of Cape Town.

MCKENZIE. B., MOLL. E.J. \& CAMPBELL. B.M. 1977. A phytosociological study of Orange Kloof. Table Mountain. South Africa. Vegeratio $34: 41-53$

MORRIS, M.I. 1981. The variation of components in the radiation balance over different finbos vegetation topes. M.A. thesis, University of Cape Town.

MUELLER-DOMBOIS, D. \& ELLENBERG, H. 1974. Aims and me thods of vegetation ecology. Wiley, New York.

MUIR. J. 1929. The vegetation of the Riversdale Area, Cape Province. Botanical Suney of South Africa, Memoir No. 13.

PRESTON-WHYTE, R.A. \& TYSON. P.D. 1988. The atmosphere and weather of southern Africa. Oxford University Press. Cape Town.

REBELO, A.G., COWLING, R.M., CAMPBELL, B.M. \& MEADOWS, M. 1991. Plant communities of the Riversdale Plain. South African Joumal of Botany 57: 10-28.

REYNOLDS, G.W. 1950. The aloes of South Africa. Trustees, The Aloes of South Africa Book Fund, Johannesburg.

ROURKE. J.P. 1972. Taxonomic studies on Leucospermum R.Br. Joumal of South Africun Botany. Supplementary Vol. 8.

ROURKE. J.P 1980. The proteas of southem Africa. Purnell, Cape Town SCHULZE. B.R. 1965. Climate of South Africa. Part 8: general survey. S.A. Weather Bureau 28. Govemment Printer, Pretoria.

SOIL CLASSIFICATION WORKING GROUP (SCWG) 1991. Soil classification: a taxonomic system for South Africa. Memoirs on the Agricultural Natural Resources of South Africa No. 15.

SWIFT. L.W. 1976. Algorithm for solar radiation on mountain slopes. Water Resources Reseanch 12: 108-112.

TAYLOR. H. \& VAN DER MEULEN, F. 1981. Structural and floristic classification of Cape mountain fynbos on Rooiberg, Southern Cape. Bothalia 13: 557-567.

TAYLOR. H.C. 1963. A bird's-eve view of the Cape mountain vegetation. Joumal of the Botanical Seciets of South Africa 49: 17-19.

TAYLOR. H.C. 1969. A vegetation survey of the Cape of Good Hope Nature Reserve. M.Sc. thesis, University of Cape Town.

TAYLOR. H.C. 1978. Capensis. In M.J.A. Werger. Biogeography and ecology of southem Africa. Junk, The Hague.

TAYLOR. H.C. 1984. A vegetation survey of the Cape of Good Hope Nature Reserve. II. Descriptive account. Bothalia 15: 259-291.

VAN HEERDEN. J. \& HURRY, L. 1987. Southern Africa's weather pattems: an introducton guide. Acacia Books. Pretoria.

VAN WILGEN, B.W. 1984. Fire climates in the southern and western Cape Province and their potential use in fire control and management. South African Joumal of Science 80: 358-362

WERGER. M.J.A. 1974. On concepts and techniques applied in the Zürich-Montpellier method of vegetation survey. Bothalia II $309-323$. 
WERGER M.J.A. KRUGER. F.J. \& TAYLOR. H.C. 1972. A phytosociological study of the Cape fynbos and other vegetation at Jonkershoek, Stellenbosch. Bothalia 10: 599-614.

WESTHOFF, V. \& VAN DER MAAREL. E. 1973. The Braun-Blanquet approach. In R.H. Whittaker, Ordination and classification of communities. Junk, The Hague.
WILLIAMS, I. 1982. Studies in the genera of the Diosmeae (Rutaceae) 14: a review of the genus Diosma L. Joumal of South African Botany 48: 329-407.

WILLIAMS, I. 1984. Studies in the genera of the Diosmeae (Rutaceae) 15: a review of the genus Empleurum Aiton. Journal of Sourh African Botany 50: 425-434.

\section{Errata in Bothalia 23,1: 157}

The vegetation of the southern Langeberg, Cape Province. 2. The plant communities of the Marloth Nature Reserve, by D.J. McDonald.

Replace 3rd paragraph, left column with:

The climate of the MNR is typical of the southern Langeberg since it falls within the transition zone between winter and year-round rainfall areas. The mean annual rainfall for the peaks is estimated to be $>1400$ $\mathrm{mm}$ (Dent et al. 1987). However, the climate on the south side of the range is distinctly different from that on the north side. The south slopes of the southern Langeberg experience the highest rainfall in late summer to autumn (March \& April) and late winter to spring (August, October \& November). The driest periods are early summer (December) and early winter
(June), Figure 5A. A more marked bimodality is evident in the rainfall pattern on the north slopes which are in a summer rainshadow. Here peaks in rainfall occur in autumn (April \& May) and late winter (August). The driest period extends from spring to late summer (October-March), Figure 5B. The mean summer maximum and mean winter minimum temperatures for Swellendam are $29.4^{\circ} \mathrm{C}$ (January) and $6.6^{\circ} \mathrm{C}$ (July) respectively. On the opposite side of the mountain at Weltevrede the equivalent temperatures are $30.0^{\circ} \mathrm{C}$ (February) and $2.9^{\circ} \mathrm{C}$ (July). 